Pacific

Journal of

Mathematics

\title{
TWISTED QUANTUM DRINFELD HECKE ALGEBRAS
}

\author{
DEEPAK NAIDU
}




\title{
TWISTED QUANTUM DRINFELD HECKE ALGEBRAS
}

\author{
DEEPAK NAIDU
}

\begin{abstract}
We generalize quantum Drinfeld Hecke algebras by incorporating a 2-cocycle on the associated finite group. We identify these algebras as specializations of deformations of twisted skew group algebras, giving an explicit connection to Hochschild cohomology. We classify these algebras for diagonal actions, as well as for the symmetric groups with their natural representations. Our results show that the parameter spaces for the symmetric groups in the twisted setting is smaller than in the untwisted setting.
\end{abstract}

\section{Introduction}

Drinfeld Hecke algebras were defined by V. Drinfeld [1986]. They arise as symplectic reflection algebras in the work of P. Etingof and V. Ginzburg [2002], as braided Cherednik algebras in the work of Y. Bazlov and A. Berenstein [2009], and as graded versions of affine Hecke algebras in the work of G. Lusztig [1989]. They arise in diverse areas, such as representation theory, combinatorics, and orbifold theory, and they were used by I. Gordon [2003] to prove a version of the $n$ ! conjecture for Weyl groups.

In this paper, we consider quantum and twisted analogs of Drinfeld Hecke algebras by incorporating quantum parameters as well as a 2-cocycle on the associated finite group. We simultaneously generalize twisted Drinfeld Hecke algebras and quantum Drinfeld Hecke algebras. The former was studied by S. Witherspoon [2007], and the latter was studied in [Levandovskyy and Shepler 2011] and [Naidu and Witherspoon 2011]. T. Chmutova [2005] generalized symplectic reflection algebras by incorporating a 2-cocycle on the associated finite group, and showed that such a 2-cocycle arises naturally for nonfaithful representations. Such a 2-cocycle also arises in orbifold theory, where they are known as discrete torsion [Adem and Ruan 2003; Căldăraru et al. 2004; Vafa and Witten 1995].

Let $V$ be a complex vector space with basis $v_{1}, v_{2}, \ldots, v_{n}$, and $\boldsymbol{q}:=\left(q_{i j}\right)_{1 \leq i, j \leq n}$, a tuple of nonzero scalars for which $q_{i i}=1$ and $q_{j i}=q_{i j}^{-1}$ for all $i, j$. Let $S_{q}(V)$

MSC2010: 16E40, 16S35.

Keywords: Hochschild cohomology, deformations, skew group algebras, graded Hecke algebras, symplectic reflection algebras. 
denote the quantum symmetric algebra

$$
\left.S_{q}(V):=\mathbb{C}\left\langle v_{1}, \ldots, v_{n}\right| v_{i} v_{j}=q_{i j} v_{j} v_{i} \text { for all } 1 \leq i, j \leq n\right\rangle .
$$

Let $G$ be a finite group acting linearly on $V$, and $\alpha: G \times G \rightarrow \mathbb{C}^{\times}$, a normalized 2-cocycle on $G$. Let $\kappa: V \times V \rightarrow \mathbb{C}^{\alpha} G$ be a bilinear map for which $\kappa\left(v_{i}, v_{j}\right)=$ $-q_{i j} \kappa\left(v_{j}, v_{i}\right)$ for all $1 \leq i, j \leq n$. Let $T(V)$ be the tensor algebra on $V$, and define

$$
\mathscr{H}_{\boldsymbol{q}, \kappa, \alpha}:=T(V) \#_{\alpha} G /\left(v_{i} v_{j}-q_{i j} v_{j} v_{i}-\kappa\left(v_{i}, v_{j}\right) \mid 1 \leq i, j \leq n\right),
$$

the quotient of the twisted skew group algebra $T(V) \#_{\alpha} G$ by the ideal generated by all elements of the form specified. Suppose that the action of $G$ on $V$ induces an action of $G$ on $S_{q}(V)$ by automorphisms, so we may form the twisted skew group algebra $S_{\boldsymbol{q}}(V) \#_{\alpha} G$. Assigning each $v_{i}$ degree one and each group element degree zero makes $\mathscr{H}_{\boldsymbol{q}, \kappa, \alpha}$ a filtered algebra, and makes $S_{\boldsymbol{q}}(V) \#_{\alpha} G$ a graded algebra. We call $\mathscr{H}_{\boldsymbol{q}, \kappa, \alpha}$ a twisted quantum Drinfeld Hecke algebra (over $\mathbb{C}$ ) if it satisfies the PoincaréBirkhoff-Witt condition: the associated graded algebra gr $\mathscr{H}_{\boldsymbol{q}, \kappa, \alpha}$ is isomorphic, as a graded algebra, to $S_{q}(V) \#_{\alpha} G$. The space of all maps $\kappa: V \times V \rightarrow \mathbb{C}^{\alpha} G$ for which $\mathscr{H}_{\boldsymbol{q}, \kappa, \alpha}$ is a twisted quantum Drinfeld Hecke algebra will be referred to as the parameter space.

Main results and organization. In Section 2, we use G. Bergman's Diamond Lemma [1978] to give necessary and sufficient conditions for the algebra $\mathscr{H}_{\boldsymbol{q}, \kappa, \alpha}$ to be a twisted quantum Drinfeld Hecke algebra.

In Section 3, we identify the twisted quantum Drinfeld Hecke algebras $\mathscr{H}_{\boldsymbol{q}, \kappa, \alpha}$ as specializations of particular types of deformations of the twisted skew group algebras $S_{q}(V) \#_{\alpha} G$.

Section 4 develops the homological algebra needed for the sections that follow. Specifically, this section is concerned with the computation of the degree two Hochschild cohomology of $S_{q}(V) \#_{\alpha} G$.

In Section 5, we establish a one-to-one correspondence between the subspace of constant Hochschild 2-cocycles (see Section 3 for definition) contained in $\mathrm{HH}^{2}\left(S_{\boldsymbol{q}}(V) \#_{\alpha} G\right)$ and twisted quantum Drinfeld Hecke algebras associated to the quadruple $(G, V, \boldsymbol{q}, \alpha)$. As a consequence, we show that every constant Hochschild 2-cocycle on $S_{\boldsymbol{q}}(V) \#_{\alpha} G$ lifts to a deformation of $S_{\boldsymbol{q}}(V) \#_{\alpha} G$.

In Section 6, we consider diagonal actions of $G$ on a chosen basis for $V$, and, using results from [Naidu et al. 2011], we classify the corresponding twisted quantum Drinfeld Hecke algebras.

In Section 7, we consider the symmetric groups $S_{n}, n \geq 5$, with their natural representations, with the unique nontrivial quantum parameters $q_{i j}=-1, i \neq j$, and with a cohomologically nontrivial 2-cocycle on $S_{n}$, which is unique up to coboundary. We classify the corresponding twisted quantum Drinfeld Hecke algebras. Our results 
show that the parameter space in the twisted setting is smaller than in the untwisted setting.

Throughout the paper, let $G$ denote a finite group acting linearly on a complex vector space $V$ with basis $v_{1}, v_{2}, \ldots, v_{n}$. Let $\boldsymbol{q}:=\left(q_{i j}\right)_{1 \leq i, j \leq n}$ denote a tuple of nonzero scalars for which $q_{i i}=1$ and $q_{j i}=q_{i j}^{-1}$ for all $i, j$. We work over the complex numbers $\mathbb{C}$, and all tensor products are taken over $\mathbb{C}$ unless otherwise indicated.

\section{Necessary and sufficient conditions}

In this section, we use Bergman's Diamond Lemma [1978] to give necessary and sufficient conditions for the algebra $\mathscr{H}_{\boldsymbol{q}, \kappa, \alpha}$ (defined in the introduction and recalled below) to be a twisted quantum Drinfeld Hecke algebra. First, we recall the notion of a twisted skew group algebra. Let $G$ be a finite group, and let $\alpha: G \times G \rightarrow \mathbb{C}^{\times}$ be a normalized 2-cocycle on $G$, that is,

$$
\alpha\left(g_{1}, g_{2}\right) \alpha\left(g_{1} g_{2}, g_{3}\right)=\alpha\left(g_{2}, g_{3}\right) \alpha\left(g_{1}, g_{2} g_{3}\right) \quad \text { and } \quad \alpha(g, 1)=1=\alpha(1, g)
$$

for all $g, g_{1}, g_{2}, g_{3} \in G$. Let $A$ be an algebra on which $G$ acts by automorphisms. The twisted skew group algebra $A \#_{\alpha} G$ is defined as follows. As a vector space, $A \#_{\alpha} G$ is $A \otimes \mathbb{C} G$. Multiplication on $A \#_{\alpha} G$ is determined by

$$
(a \otimes g)(b \otimes h):=\alpha(g, h) a\left({ }^{g} b\right) \otimes g h
$$

for all $a, b \in A$ and all $g, h \in G$, where a left superscript denotes the action of the group element. The 2-cocycle condition on $\alpha$ ensures that $A \#_{\alpha} G$ is an associative algebra. Note that $A$ is a subalgebra of $A \#_{\alpha} G$ via the isomorphism $A \stackrel{\sim}{\rightarrow} A \otimes 1$, and the twisted group algebra $\mathbb{C}^{\alpha} G$ is a subalgebra of $A \#_{\alpha} G$ via the isomorphism $\mathbb{C}^{\alpha} G \stackrel{\sim}{\rightarrow} 1 \otimes \mathbb{C}^{\alpha} G$. The image of a group element $g$ in the twisted group algebra $\mathbb{C}^{\alpha} G$ is denoted by $t_{g}$. To shorten notation, we write the element $a \otimes g$ of $A \#_{\alpha} G$ by $a t_{g}$. Since $\alpha$ is assumed to be normalized, $t_{1}$ is the multiplicative identity for $A \#_{\alpha} G$. For all $g \in G$, we have

$$
\left(t_{g}\right)^{-1}=\alpha^{-1}\left(g, g^{-1}\right) t_{g^{-1}}=\alpha^{-1}\left(g^{-1}, g\right) t_{g^{-1}} .
$$

Suppose $G$ acts linearly on a complex vector space $V$ with basis $v_{1}, v_{2}, \ldots, v_{n}$, and let $\boldsymbol{q}:=\left(q_{i j}\right)_{1 \leq i, j \leq n}$ denote a tuple of nonzero scalars for which $q_{i i}=1$ and $q_{j i}=q_{i j}^{-1}$ for all $i, j$. For each group element $g \in G$, let $g_{k}^{i}$ denote the scalar determined by the equation

$$
{ }^{g} v_{i}=\sum_{k=1}^{n} g_{k}^{i} v_{k},
$$

and define the quantum $(i, j, k, l)$-minor determinant of $g$ as

$$
\operatorname{det}_{i j k l}(g):=g_{l}^{j} g_{k}^{i}-q_{j i} g_{l}^{i} g_{k}^{j} \text {. }
$$


The following lemma is used in the proof of Theorem 2.2.

Lemma 2.1. Suppose that the action of $G$ on $V$ extends to an action on $S_{q}(V)$ by automorphisms, and let $g \in G$. We have:

(i) $q_{l k} \operatorname{det}_{i j k l}(g)=-\operatorname{det}_{i j l k}(g)$ for all $i, j, k, l$.

(ii) For each $i, j$, if $q_{i j} \neq 1$, then $g_{k}^{i} g_{k}^{j}=0$ for all $k$.

Proof. For a proof of (i), see [Levandovskyy and Shepler 2011, Lemma 3.2]. Part (ii) follows from the assumption that $G$ acts on $S_{q}(V)$ by automorphisms and that $q_{i j} \neq 1$ : we have ${ }^{g} v_{i}{ }^{g} v_{j}=q_{i j}{ }^{g} v_{j}{ }^{g} v_{i}$, and so $\left(\sum_{k=1}^{n} g_{k}^{i} v_{k}\right)\left(\sum_{l=1}^{n} g_{l}^{j} v_{l}\right)=$ $q_{i j}\left(\sum_{k=1}^{n} g_{k}^{j} v_{k}\right)\left(\sum_{l=1}^{n} g_{l}^{i} v_{l}\right)$. Equating coefficients of $v_{k}^{2}$ yields $g_{k}^{i} g_{k}^{j}=q_{i j} g_{k}^{i} g_{k}^{j}$, and since $q_{i j} \neq 1$, we get $g_{k}^{i} g_{k}^{j}=0$.

Let $\kappa: V \times V \rightarrow \mathbb{C}^{\alpha} G$ be a bilinear map for which $\kappa\left(v_{i}, v_{j}\right)=-q_{i j} \kappa\left(v_{j}, v_{i}\right)$ for all $1 \leq i, j \leq n$. For each $g \in G$, let $\kappa_{g}: V \times V \rightarrow \mathbb{C}$ be the function determined by the condition

$$
\kappa(v, w)=\sum_{g \in G} \kappa_{g}(v, w) t_{g} \quad \text { for all } v, w \in V .
$$

The condition $\kappa\left(v_{i}, v_{j}\right)=-q_{i j} \kappa\left(v_{j}, v_{i}\right)$ implies that $\kappa_{g}\left(v_{i}, v_{j}\right)=-q_{i j} \kappa_{g}\left(v_{j}, v_{i}\right)$ for all $g \in G$.

Recall that the algebra

$$
\mathscr{H}_{\boldsymbol{q}, \kappa, \alpha}:=T(V) \#_{\alpha} G /\left(v_{i} v_{j}-q_{i j} v_{j} v_{i}-\kappa\left(v_{i}, v_{j}\right) \mid 1 \leq i, j \leq n\right)
$$

is called a twisted quantum Drinfeld Hecke algebra if it satisfies the PoincaréBirkhoff-Witt condition: $\mathrm{gr} \mathscr{H}_{\boldsymbol{q}, \kappa, \alpha} \cong S_{\boldsymbol{q}}(V) \#_{\alpha} G$, as graded algebras. This is equivalent to the condition that the set $\left\{v_{1}^{m_{1}} v_{2}^{m_{2}} \cdots v_{n}^{m_{n}} t_{g} \mid m_{i} \geq 0, g \in G\right\}$ is a $\mathbb{C}$-basis for $\mathscr{H}_{\boldsymbol{q}, \kappa, \alpha}$.

In the proof of the next theorem, we assume familiarity with, and will freely use, terminology from [Bergman 1978] (for example, "reduction system").

Theorem 2.2. The algebra $\mathscr{H}_{\boldsymbol{q}, \kappa, \alpha}$ is a twisted quantum Drinfeld Hecke algebra if and only if the following conditions hold.

(1) For all $g, h \in G$ and $1 \leq i<j \leq n$,

$$
\frac{\alpha(h, g)}{\alpha\left(h g h^{-1}, h\right)} \kappa_{g}\left(v_{j}, v_{i}\right)=\sum_{k<l} \operatorname{det}_{i j k l}(h) \kappa_{h g h^{-1}}\left(v_{l}, v_{k}\right) .
$$

(2) For all $g \in G$ and $1 \leq i<j<k \leq n$,

$\kappa_{g}\left(v_{k}, v_{j}\right)\left({ }^{g} v_{i}-q_{j i} q_{k i} v_{i}\right)+\kappa_{g}\left(v_{k}, v_{i}\right)\left(q_{k j} v_{j}-q_{j i}{ }^{g} v_{j}\right)+\kappa_{g}\left(v_{j}, v_{i}\right)\left(q_{k j} q_{k i}{ }^{g} v_{k}-v_{k}\right)=0$. 
Proof. We begin by expressing the algebra $\mathscr{H}_{\boldsymbol{q}, \kappa, \alpha}$ as a quotient of a free associative $\mathbb{C}$-algebra. Let $X=\left\{v_{1}, v_{2}, \ldots, v_{n}\right\} \cup\left\{t_{g} \mid g \in G\right\}$, and let $\mathbb{C}\langle X\rangle$ be the free associative $\mathbb{C}$-algebra generated by $X$. Consider the reduction system

$S=\left\{\left(t_{g} v_{i},{ }^{g} v_{i} t_{g}\right),\left(t_{g} t_{h}, \alpha(g, h) t_{g h}\right),\left(v_{j} v_{i}, q_{j i} v_{i} v_{j}+\kappa\left(v_{j}, v_{i}\right)\right) \mid g, h \in G, 1 \leq i<j \leq n\right\}$ for $\mathbb{C}\langle X\rangle$. Let $I$ be the ideal of $\mathbb{C}\langle X\rangle$ generated by the elements $t_{g} v_{i}-{ }^{g} v_{i} t_{g}, \quad t_{g} t_{h}-\alpha(g, h) t_{g h}, \quad v_{j} v_{i}-q_{j i} v_{i} v_{j}-\kappa\left(v_{j}, v_{i}\right), \quad g, h \in G, 1 \leq i<j \leq n$. In what follows, we use the Diamond Lemma [Bergman 1978] to show that the set

$$
\left\{v_{1}^{m_{1}} v_{2}^{m_{2}} \cdots v_{n}^{m_{n}} t_{g} \mid m_{i} \geq 0, g \in G\right\}
$$

is a $\mathbb{C}$-basis for $\mathbb{C}\langle X\rangle / I$ if and only if the two conditions in the statement of the theorem hold.

Define a partial order $\leq$ on the free semigroup $\langle X\rangle$ as follow: First, we declare that $v_{1}<v_{2}<\cdots<v_{n}<g$ for all $g \in G$, and then we set $A<B$ if

(i) $A$ is of smaller length than $B$, or

(ii) $A$ and $B$ have the same length but $A$ is less than $B$ relative to the lexicographical order.

Then $\leq$ is a semigroup partial order on $\langle X\rangle$, compatible with the reduction system $S$, and having the descending chain condition. Thus the hypothesis of the Diamond Lemma holds.

Observe that the set $\langle X\rangle_{\text {irr }}$ of irreducible elements of $\langle X\rangle$ is precisely the alleged $\mathbb{C}$-basis for $\mathbb{C}\langle X\rangle / I$. That is,

$$
\langle X\rangle_{\text {irr }}=\left\{v_{1}^{m_{1}} v_{2}^{m_{2}} \cdots v_{n}^{m_{n}} t_{g} \mid m_{i} \geq 0, g \in G\right\} .
$$

In what follows, we show that all ambiguities of $S$ are resolvable if and only if the two conditions in the statement of the theorem hold. The theorem will then follow by the Diamond Lemma. There are no inclusion ambiguities, but there exist overlap ambiguities, and these correspond to the monomials

$$
t_{g} t_{h} t_{k}, \quad t_{g} t_{h} v_{i}, \quad t_{h} v_{j} v_{i}, \quad v_{k} v_{j} v_{i}, \quad \text { where } 1 \leq i<j<k \leq n, g, h \in G .
$$

Associativity of the multiplication in the twisted group algebra $\mathbb{C}^{\alpha} G$ implies that the ambiguities corresponding to the monomials $t_{g} t_{h} t_{k}$ are resolvable. The equality ${ }^{g h} v_{i}={ }^{g}\left({ }^{h} v_{i}\right)$ implies that the ambiguities corresponding to the monomials $t_{g} t_{h} v_{i}$ are resolvable. Next, we show that the ambiguities corresponding to the monomials $t_{h} v_{j} v_{i}$ are resolvable if and only if condition (1) in the statement of the theorem holds. Applying a reduction to the factor $v_{j} v_{i}$ in $t_{h} v_{j} v_{i}$, we get

$$
q_{j i} t_{h} v_{i} v_{j}+t_{h} \kappa\left(v_{j}, v_{i}\right) \text {. }
$$


Applying a reduction to the factor $t_{h} v_{i}$ and then to the resulting factor $t_{h} v_{j}$ gives

$$
\begin{aligned}
& q_{j i}{ }^{h} v_{i}{ }^{h} v_{j} t_{h}+t_{h} \kappa\left(v_{j}, v_{i}\right) \\
& \quad=q_{j i}\left(\sum_{l=1}^{n} h_{l}^{i} v_{l}\right)\left(\sum_{k=1}^{n} h_{k}^{j} v_{k}\right) t_{h}+t_{h} \kappa\left(v_{j}, v_{i}\right) \\
& \quad=q_{j i} \sum_{l<k} h_{l}^{i} h_{k}^{j} v_{l} v_{k} t_{h}+q_{j i} \sum_{k<l} h_{l}^{i} h_{k}^{j} v_{l} v_{k} t_{h}+q_{j i} \sum_{k=1}^{n} h_{k}^{i} h_{k}^{j} v_{k}^{2} t_{h}+t_{h} \kappa\left(v_{j}, v_{i}\right) .
\end{aligned}
$$

Applying a reduction to the factor $v_{l} v_{k}$ in the second summation above yields

$$
\begin{aligned}
q_{j i} \sum_{l<k} h_{l}^{i} h_{k}^{j} v_{l} v_{k} t_{h}+q_{j i} & \sum_{k<l} h_{l}^{i} h_{k}^{j} q_{l k} v_{k} v_{l} t_{h} \\
& +q_{j i} \sum_{k<l} h_{l}^{i} h_{k}^{j} \kappa\left(v_{l}, v_{k}\right) t_{h}+q_{j i} \sum_{k=1}^{n} h_{k}^{i} h_{k}^{j} v_{k}^{2} t_{h}+t_{h} \kappa\left(v_{j}, v_{i}\right) .
\end{aligned}
$$

Combining the first two summations, expanding $\kappa\left(v_{l}, v_{k}\right)$ and $\kappa\left(v_{j}, v_{i}\right)$, and then applying reductions to each term in $\kappa\left(v_{l}, v_{k}\right) t_{h}$ and to each term in $t_{h} \kappa\left(v_{j}, v_{i}\right)$ gives

$$
\begin{aligned}
q_{j i} \sum_{k<l}\left(h_{k}^{i} h_{l}^{j}+q_{l k} h_{l}^{i} h_{k}^{j}\right) v_{k} v_{l} t_{h}+q_{j i} \sum_{k=1}^{n} h_{k}^{i} h_{k}^{j} v_{k}^{2} t_{h} \\
\quad+q_{j i} \sum_{g \in G}\left(\alpha(g, h) \sum_{k<l} h_{l}^{i} h_{k}^{j} \kappa_{g}\left(v_{l}, v_{k}\right)\right) t_{g h}+\sum_{g \in G} \alpha(h, g) \kappa_{g}\left(v_{j}, v_{i}\right) t_{h g} \\
=q_{j i} \sum_{k<l}\left(h_{k}^{i} h_{l}^{j}+q_{l k} h_{l}^{i} h_{k}^{j}\right) v_{k} v_{l} t_{h}+q_{j i} \sum_{k=1}^{n} h_{k}^{i} h_{k}^{j} v_{k}^{2} t_{h} \\
\quad+\sum_{g \in G}\left(\alpha\left(h g h^{-1}, h\right) q_{j i} \sum_{k<l} h_{l}^{i} h_{k}^{j} \kappa_{h g h^{-1}}\left(v_{l}, v_{k}\right)+\alpha(h, g) \kappa_{g}\left(v_{j}, v_{i}\right)\right) t_{h g} .
\end{aligned}
$$

Next, we apply to $t_{h} v_{j} v_{i}$ a reduction different from the one in the computation above: Applying a reduction to the factor $t_{h} v_{j}$ in $t_{h} v_{j} v_{i}$, and then to the resulting factor $t_{h} v_{i}$, we get

$$
\begin{aligned}
{ }^{h} v_{j}{ }^{h} v_{i} t_{h} & =\left(\sum_{l=1}^{n} h_{l}^{j} v_{l}\right)\left(\sum_{k=1}^{n} h_{k}^{i} v_{k}\right) t_{h} \\
& =\sum_{l<k} h_{l}^{j} h_{k}^{i} v_{l} v_{k} t_{h}+\sum_{k<l} h_{l}^{j} h_{k}^{i} v_{l} v_{k} t_{h}+\sum_{k=1}^{n} h_{k}^{j} h_{k}^{i} v_{k}^{2} t_{h} .
\end{aligned}
$$

Applying a reduction to the factor $v_{l} v_{k}$ in the second summation above yields

$$
\sum_{l<k} h_{l}^{j} h_{k}^{i} v_{l} v_{k} t_{h}+\sum_{k<l} q_{l k} h_{l}^{j} h_{k}^{i} v_{k} v_{l} t_{h}+\sum_{k<l} h_{l}^{j} h_{k}^{i} \kappa\left(v_{l}, v_{k}\right) t_{h}+\sum_{k=1}^{n} h_{k}^{j} h_{k}^{i} v_{k}^{2} t_{h} .
$$


Combining the first two summations, expanding $\kappa\left(v_{l}, v_{k}\right)$, and then applying a reduction to each term in $\kappa\left(v_{l}, v_{k}\right) t_{h}$ gives

$$
\begin{gathered}
\sum_{k<l}\left(h_{k}^{j} h_{l}^{i}+q_{l k} h_{l}^{j} h_{k}^{i}\right) v_{k} v_{l} t_{h}+\sum_{k=1}^{n} h_{k}^{j} h_{k}^{i} v_{k}^{2} t_{h}+\sum_{g \in G}\left(\alpha(g, h) \sum_{k<l} h_{l}^{j} h_{k}^{i} \kappa_{g}\left(v_{l}, v_{k}\right)\right) t_{g h} \\
=\sum_{k<l}\left(h_{k}^{j} h_{l}^{i}+q_{l k} h_{l}^{j} h_{k}^{i}\right) v_{k} v_{l} t_{h}+\sum_{k=1}^{n} h_{k}^{j} h_{k}^{i} v_{k}^{2} t_{h} \\
+\sum_{g \in G}\left(\alpha\left(h g h^{-1}, h\right) \sum_{k<l} h_{l}^{j} h_{k}^{i} \kappa_{h g h^{-1}}\left(v_{l}, v_{k}\right)\right) t_{h g} .
\end{gathered}
$$

By equating coefficients, we see that the final expressions in the previous two computations are equal if and only if

(a) $q_{j i} h_{k}^{i} h_{l}^{j}+q_{j i} q_{l k} h_{l}^{i} h_{k}^{j}=h_{k}^{j} h_{l}^{i}+q_{l k} h_{l}^{j} h_{k}^{i}$ for all $k<l$,

(b) $q_{j i} h_{k}^{i} h_{k}^{j}=h_{k}^{i} h_{k}^{j}$ for all $k$, and

(c) for all $g \in G$, we have

$$
\begin{aligned}
\alpha\left(h g h^{-1}, h\right) q_{j i} \sum_{k<l} h_{l}^{i} h_{k}^{j} \kappa_{h g h^{-1}}\left(v_{l}, v_{k}\right)+\alpha & (h, g) \kappa_{g}\left(v_{j}, v_{i}\right) \\
& =\alpha\left(h g h^{-1}, h\right) \sum_{k<l} h_{l}^{j} h_{k}^{i} \kappa_{h g h^{-1}}\left(v_{l}, v_{k}\right) .
\end{aligned}
$$

Conditions (a) and (b) follow from Lemma 2.1(i) and (ii), respectively. The equation in (c) is equivalent to condition (1) in the statement of the theorem.

Lastly, we show that the ambiguities corresponding to the monomials $v_{k} v_{j} v_{i}$ are resolvable if and only if condition (2) in the statement of the theorem holds. Applying a reduction to the factor $v_{k} v_{j}$ in $v_{k} v_{j} v_{i}$, we get

$$
q_{k j} v_{j} v_{k} v_{i}+\kappa\left(v_{k}, v_{j}\right) v_{i} \text {. }
$$

Applying a reduction to the factor $v_{k} v_{i}$ gives

$$
q_{k j} q_{k i} v_{j} v_{i} v_{k}+q_{k j} v_{j} \kappa\left(v_{k}, v_{i}\right)+\kappa\left(v_{k}, v_{j}\right) v_{i} .
$$

Applying a reduction to the factor $v_{j} v_{i}$ yields

$$
q_{k j} q_{k i} q_{j i} v_{i} v_{j} v_{k}+q_{k j} q_{k i} \kappa\left(v_{j}, v_{i}\right) v_{k}+q_{k j} v_{j} \kappa\left(v_{k}, v_{i}\right)+\kappa\left(v_{k}, v_{j}\right) v_{i} .
$$

Expanding $\kappa\left(v_{j}, v_{i}\right), \kappa\left(v_{k}, v_{i}\right)$, and $\kappa\left(v_{k}, v_{j}\right)$, applying reductions to each term in $\kappa\left(v_{j}, v_{i}\right) v_{k}$ and to each term in $\kappa\left(v_{k}, v_{j}\right) v_{i}$, and then rearranging gives

$$
q_{k j} q_{k i} q_{j i} v_{i} v_{j} v_{k}+\sum_{g \in G}\left(\kappa_{g}\left(v_{k}, v_{j}\right)^{g} v_{i}+q_{k j} \kappa_{g}\left(v_{k}, v_{i}\right) v_{j}+q_{k j} q_{k i} \kappa_{g}\left(v_{j}, v_{i}\right)^{g} v_{k}\right) t_{g} .
$$

Next, we apply to $v_{k} v_{j} v_{i}$ a reduction different from the one in the computation above: Applying a reduction to the factor $v_{j} v_{i}$ in $v_{k} v_{j} v_{i}$, we get

$$
q_{j i} v_{k} v_{i} v_{j}+v_{k} \kappa\left(v_{j}, v_{i}\right) \text {. }
$$


Applying a reduction to the factor $v_{k} v_{i}$ gives

$$
q_{j i} q_{k i} v_{i} v_{k} v_{j}+q_{j i} \kappa\left(v_{k}, v_{i}\right) v_{j}+v_{k} \kappa\left(v_{j}, v_{i}\right) .
$$

Applying a reduction to the factor $v_{k} v_{j}$ yields

$$
q_{j i} q_{k i} q_{k j} v_{i} v_{j} v_{k}+q_{j i} q_{k i} v_{i} \kappa\left(v_{k}, v_{j}\right)+q_{j i} \kappa\left(v_{k}, v_{i}\right) v_{j}+v_{k} \kappa\left(v_{j}, v_{i}\right) .
$$

Expanding $\kappa\left(v_{k}, v_{j}\right), \kappa\left(v_{k}, v_{i}\right)$, and $\kappa\left(v_{j}, v_{i}\right)$, and then applying reductions to each term in $\kappa\left(v_{k}, v_{i}\right) v_{j}$ gives

$$
q_{j i} q_{k i} q_{k j} v_{i} v_{j} v_{k}+\sum_{g \in G}\left(q_{j i} q_{k i} \kappa_{g}\left(v_{k}, v_{j}\right) v_{i}+q_{j i} \kappa_{g}\left(v_{k}, v_{i}\right)^{g} v_{j}+\kappa_{g}\left(v_{j}, v_{i}\right) v_{k}\right) t_{g} .
$$

The final expressions in the two computations above are equal if and only if condition (2) in the statement of the theorem holds. This finishes the proof.

\section{Deformations}

The primary goal of this section is to show that the twisted quantum Drinfeld Hecke algebras $\mathscr{H}_{\boldsymbol{q}, \kappa, \alpha}$ are isomorphic to specializations of particular types of deformations of the twisted skew group algebras $S_{q}(V) \#_{\alpha} G$.

Let $\hbar$ denote an indeterminate. Recall that, for a $\mathbb{C}$-algebra $A$, a deformation of $A$ over $\mathbb{C}[\hbar]$ is an associative $\mathbb{C}[\hbar]$-algebra whose underlying vector space is $A[\hbar]=\mathbb{C}[\hbar] \otimes A$, and which reduces modulo $\hbar$ to the original algebra $A$. Thus the multiplication $\mu$ on $A[\hbar]$ is determined by

$$
\mu(a, b)=\mu_{0}(a, b)+\mu_{1}(a, b) \hbar+\mu_{2}(a, b) \hbar^{2}+\cdots
$$

for all $a, b \in A$, where $\mu_{0}(a, b)$ is the product in $A$, the $\mu_{i}: A \times A \rightarrow A$ are $\mathbb{C}$-bilinear maps extended to be bilinear over $\mathbb{C}[\hbar]$, and for each pair $(a, b)$ the sum above is finite. A consequence of associativity of $\mu$ is that $\mu_{1}$ is a Hochschild 2-cocycle, that is,

$$
a \mu_{1}(b, c)+\mu_{1}(a, b c)=\mu_{1}(a b, c)+\mu_{1}(a, b) c
$$

for all $a, b, c \in A$.

In order to see that the twisted quantum Drinfeld Hecke algebras $\mathscr{H}_{\boldsymbol{q}, \kappa, \alpha}$ may be realized as specializations of deformations of $S_{q}(V) \#_{\alpha} G$, we define the algebra

$$
\mathcal{H}_{\boldsymbol{q}, \kappa, \alpha, \hbar}:=\left(T(V) \#_{\alpha} G\right)[\hbar] /\left(v_{i} v_{j}-q_{i j} v_{j} v_{i}-\kappa\left(v_{i}, v_{j}\right) \hbar \mid 1 \leq i, j \leq n\right) .
$$

Assigning $\hbar$ degree zero, each $v_{i}$ degree one, and each $t_{g}(g \in G)$ degree zero, we see that $\mathscr{H}_{\boldsymbol{q}, \kappa, \alpha, \hbar}$ is a filtered algebra, and that $\left(S_{\boldsymbol{q}}(V) \#_{\alpha} G\right)[\hbar]$ is a graded algebra. We call the algebra $\mathscr{H}_{\boldsymbol{q}, \kappa, \alpha, \hbar}$ a twisted quantum Drinfeld Hecke algebra over $\mathbb{C}[\hbar]$ if gr $\mathscr{H}_{\boldsymbol{q}, \kappa, \alpha, \hbar} \cong\left(S_{\boldsymbol{q}}(V) \#_{\alpha} G\right)[\hbar]$, as graded algebras. Specializing a twisted quantum 
Drinfeld Hecke algebra over $\mathbb{C}[\hbar]$ to $\hbar=1$ yields the twisted quantum Drinfeld Hecke algebra over $\mathbb{C}$, as defined earlier.

In the following theorem, by the degree of $\mu_{i}$ we mean its degree as a function between graded algebras.

Theorem 3.2. Every twisted quantum Drinfeld Hecke algebra $\mathscr{H}_{q, \kappa, \alpha, \hbar}$ over $\mathbb{C}[\hbar]$ is isomorphic to some deformation $\mu=\mu_{0}+\mu_{1} \hbar+\mu_{2} \hbar^{2}+\cdots$ of $S_{q}(V) \#_{\alpha} G$ over $\mathbb{C}[\hbar]$ with $\operatorname{deg} \mu_{i}=-2 i$ for all $i \geq 1$.

Proof. Suppose that $\mathscr{H}_{\boldsymbol{q}, \kappa, \alpha, \hbar}$ is a twisted quantum Drinfeld Hecke algebra over $\mathbb{C}[\hbar]$. Consider the natural projection $T(V) \#_{\alpha} G \rightarrow S_{q}(V) \#_{\alpha} G$, and let $s: S_{q}(V) \#_{\alpha} G \rightarrow$ $T(V) \#_{\alpha} G$ be the $\mathbb{C}$-linear section determined by the ordering $v_{1}, v_{2}, \ldots, v_{n}$ of the basis of $V$. For example, $s\left(v_{2} v_{1}^{2} t_{g}\right)=q_{21}^{2} v_{1}^{2} v_{2} t_{g}$.

Extend $s$ to a $\mathbb{C}[\hbar]$-linear map $\tilde{s}:\left(S_{q}(V) \#_{\alpha} G\right)[\hbar] \rightarrow\left(T(V) \#_{\alpha} G\right)[\hbar]$, and let $p$ denote the natural projection from $\left(T(V) \#_{\alpha} G\right)[\hbar]$ to $\mathscr{H}_{\boldsymbol{q}, \kappa, \alpha, \hbar}$. Since $\mathscr{H}_{\boldsymbol{q}, \kappa, \alpha, \hbar}$ is a twisted quantum Drinfeld Hecke algebra over $\mathbb{C}[\hbar]$, the composition $f:=p \circ \tilde{s}$ is an isomorphism of $\mathbb{C}[\hbar]$-modules.

Next, define a $\mathbb{C}[\hbar]$-bilinear multiplication $\mu$ on $\left(S_{\boldsymbol{q}}(V) \#_{\alpha} G\right)[\hbar]$ by

$$
\mu:=f^{-1} \circ \text { mult } \circ(f \times f),
$$

where mult is the multiplication map in $\mathscr{H}_{\boldsymbol{q}, \kappa, \alpha, \hbar}$. Since $\mu$ is $\mathbb{C}[\hbar]$-bilinear, it must necessarily be a power series

$$
\mu=\mu_{0}+\mu_{1} \hbar+\mu_{2} \hbar+\cdots,
$$

where the $\mu_{i}$ are $\mathbb{C}$-bilinear maps from $\left(S_{\boldsymbol{q}}(V) \#_{\alpha} G\right) \times\left(S_{\boldsymbol{q}}(V) \#_{\alpha} G\right)$ to $S_{\boldsymbol{q}}(V) \#_{\alpha} G$. Note that, by definition of $f$, the map $\mu_{0}$ is precisely the multiplication map in $S_{q}(V) \#_{\alpha} G$, and so $\mu$ is a deformation $S_{q}(V) \#_{\alpha} G$ over $\mathbb{C}[\hbar]$. By definition, the map $f$ is an isomorphism between the $\mathbb{C}[\hbar]$-algebras $\left(S_{\boldsymbol{q}}(V) \#_{\alpha} G[\hbar], \mu\right)$ and $\mathscr{H}_{\boldsymbol{q}, \kappa, \alpha, \hbar}$, proving that $\mathscr{H}_{\boldsymbol{q}, \kappa, \alpha, \hbar}$ is isomorphic to a deformation of $S_{\boldsymbol{q}}(V) \#_{\alpha} G$ over $\mathbb{C}[\hbar]$.

Finally we prove the degree condition on the $\mu_{i}$. Given elements

$$
a=v_{1}^{\beta_{1}} v_{2}^{\beta_{2}} \cdots v_{n}^{\beta_{n}} t_{g} \quad \text { and } \quad b=v_{1}^{\gamma_{1}} v_{2}^{\gamma_{2}} \cdots v_{n}^{\gamma_{n}} t_{h}
$$

in $S_{q}(V) \#_{\alpha} G$, to find $\mu_{1}(a, b), \mu_{2}(a, b), \ldots$, we must put the product $f(a) f(b) \in$ $\mathscr{H}_{\boldsymbol{q}, \kappa, \alpha, \hbar}$ in the normal form by repeatedly applying the relations defining $\mathscr{H}_{\boldsymbol{q}, \kappa, \alpha, \hbar}$. Induction on the degree $\sum_{k=1}^{n} \beta_{k}+\gamma_{k}$ of $a b$ implies that $\operatorname{deg} \mu_{i}=-2 i$ for all $i \geq 1$, as claimed.

Lemma 3.3. The algebra $\mathscr{H}_{\boldsymbol{q}, \kappa, \alpha}$ is a twisted quantum Drinfeld Hecke algebra over $\mathbb{C}$ if and only if $\mathcal{H}_{\boldsymbol{q}, \kappa, \alpha, \hbar}$ is a twisted quantum Drinfeld Hecke algebra over $\mathbb{C}[\hbar]$.

Proof. The proof given for $\mathscr{H}_{\boldsymbol{q}, \kappa, \alpha}$ in Theorem 2.2 generalizes for $\mathscr{H}_{\boldsymbol{q}, \kappa, \alpha, \hbar}$ by extending scalars to $\mathbb{C}[\hbar]$. That is, $\mathscr{H}_{\boldsymbol{q}, \kappa, \alpha, \hbar}$ is a twisted quantum Drinfeld Hecke algebra over $\mathbb{C}[\hbar]$ if and only if the two conditions in Theorem 2.2 hold. 
Corollary 3.4. Every twisted quantum Drinfeld Hecke algebra $\mathscr{H}_{\boldsymbol{q}, \kappa, \alpha}$ is isomorphic to a specialization of a deformation $\mu=\mu_{0}+\mu_{1} \hbar+\mu_{2} \hbar^{2}+\cdots$ of $S_{q}(V) \#_{\alpha} G$ over $\mathbb{C}[\hbar]$ with $\operatorname{deg} \mu_{i}=-2 i$ for all $i \geq 1$.

A Hochschild 2-cocycle on $S_{q}(V) \#_{\alpha} G$ is said to be constant if it is of degree -2 as a function between graded algebras. In the next section, it is shown that such 2-cocycles correspond to certain constant polynomials, justifying the choice of terminology.

Proposition 3.5. Let $\mathscr{H}_{\boldsymbol{q}, \kappa, \alpha}$ be a twisted quantum Drinfeld Hecke algebra. The map $\kappa: V \times V \rightarrow \mathbb{C}^{\alpha} G$ is equal to the quantum skew-symmetrization of some constant Hochschild 2-cocycle $\mu_{1}$ on $S_{q}(V) \#_{\alpha} G$, that is,

$$
\kappa\left(v_{i}, v_{j}\right)=\mu_{1}\left(v_{i}, v_{j}\right)-q_{i j} \mu_{1}\left(v_{j}, v_{i}\right)
$$

for all $i, j$.

Proof. By Lemma 3.3, $\mathscr{H}_{\boldsymbol{q}, \kappa, \alpha, \hbar}$ is a twisted quantum Drinfeld Hecke algebra over $\mathbb{C}[\hbar]$. By Theorem 3.2, associated to $\mathscr{H}_{\boldsymbol{q}, \kappa, \alpha, \hbar}$ is a deformation $\mu=\mu_{0}+\mu_{1} \hbar+$ $\mu_{2} \hbar^{2}+\cdots$ of $S_{q}(V) \#_{\alpha} G$ over $\mathbb{C}[\hbar]$ with $\operatorname{deg} \mu_{i}=-2 i$ for all $i \geq 1$. Note that $\mu_{1}$ is a constant Hochschild 2-cocycle on $S_{q}(V) \#_{\alpha} G$. We claim that $\kappa$ is equal to the quantum skew-symmetrization of $\mu_{1}$.

Let $f$ be the map defined in the proof of Theorem 3.2. For any two monomials $a, b \in S_{q}(V) \#_{\alpha} G$, the value of $\mu_{1}(a, b)$ is determined by writing the product $f(a) f(b) \in \mathscr{H}_{\boldsymbol{q}, \kappa, \alpha, \hbar}$ in the normal form by repeatedly applying the relations defining $\mathscr{H}_{\boldsymbol{q}, \kappa, \alpha, \hbar}$. If $i \leq j$, the product $f\left(v_{i}\right) f\left(v_{j}\right)=v_{i} v_{j}$ is already in the desired form, so $\mu_{1}\left(v_{i}, v_{j}\right)=0$. If $i>j$, we write $v_{i} v_{j} \longrightarrow q_{i j} v_{j} v_{i}+\kappa\left(v_{i}, v_{j}\right) \hbar$, and so $\kappa\left(v_{i}, v_{j}\right)=$ $\mu_{1}\left(v_{i}, v_{j}\right)$. If $i \leq j$, we have $\kappa\left(v_{i}, v_{j}\right)=-q_{i j} \kappa\left(v_{j}, v_{i}\right)=-q_{i j} \mu_{1}\left(v_{j}, v_{i}\right)$. Thus $\kappa\left(v_{i}, v_{j}\right)=\mu_{1}\left(v_{i}, v_{j}\right)-q_{i j} \mu_{1}\left(v_{j}, v_{i}\right)$ for all $i, j$.

The proof of the following theorem is a generalization of [Naidu and Witherspoon 2011, Theorem 2.2]; see also [Witherspoon 2007, Theorem 3.2].

Theorem 3.6. Every deformation $\mu=\mu_{0}+\mu_{1} \hbar+\mu_{2} \hbar^{2}+\cdots$ of $S_{\boldsymbol{q}}(V) \#_{\alpha} G$ over $\mathbb{C}[\hbar]$ with $\operatorname{deg} \mu_{i}=-2 i$ for all $i \geq 1$ is isomorphic to some twisted quantum Drinfeld Hecke algebra over $\mathbb{C}[\hbar]$.

Proof. Suppose that $\mu=\mu_{0}+\mu_{1} \hbar+\mu_{2} \hbar^{2}+\cdots$ is a deformation of $S_{q}(V) \#_{\alpha} G$ over $\mathbb{C}[\hbar]$ with $\operatorname{deg} \mu_{i}=-2 i$ for all $i \geq 1$. In what follows, we identity $T(V) \#_{\alpha} G$ with the free associative $\mathbb{C}$-algebra generated by the set $\left\{v_{1}, v_{2}, \ldots, v_{n}\right\} \cup\left\{t_{g} \mid g \in G\right\}$ subject to the relations $t_{g} v_{i}={ }^{g} v_{i} t_{g}$ and $t_{g} t_{h}=\alpha(g, h) t_{g h}$ for all $i \in\{1,2, \ldots, n\}$ and all $g, h \in G$. Define a map $\phi:\left(T(V) \#_{\alpha} G\right)[\hbar] \rightarrow\left(S_{q}(V) \#_{\alpha} G\right)[\hbar]$ as follows. First, set $\phi\left(v_{i}\right)=v_{i}$ and $\phi\left(t_{g}\right)=t_{g}$ for all $i \in\{1,2, \ldots, n\}$ and all $g \in G$. Since $\operatorname{deg} \mu_{k}=-2 k$ for all $k \geq 1$, we have

$$
\mu_{k}\left(\mathbb{C}^{\alpha} G, \mathbb{C}^{\alpha} G\right)=\mu_{k}\left(\mathbb{C}^{\alpha} G, V\right)=\mu_{k}\left(V, \mathbb{C}^{\alpha} G\right)=0
$$


for all $k \geq 1$. This implies that the relations $t_{g} v_{i}={ }^{g} v_{i} t_{g}$ and $t_{g} t_{h}=\alpha(g, h) t_{g h}$ hold in the algebra $\left(\left(S_{q}(V) \#_{\alpha} G\right)[\hbar], \mu\right)$, and so we obtain a $\mathbb{C}$-algebra homomorphism on $T(V) \#_{\alpha} G$, which extends to a $\mathbb{C}[\hbar]$-algebra homomorphism $\phi$ from $\left(T(V) \#_{\alpha} G\right)[\hbar]$ to $\left(S_{q}(V) \#_{\alpha} G\right)[\hbar]$, where the algebra structure on the latter is given by $\mu$.

Next, we show that $\phi$ is surjective. It is enough to show that each monomial $v_{i_{1}} \cdots v_{i_{m}} t_{g}$ is in the image of $\phi$. The proof is by induction on the degree of the monomial. Suppose that all monomials of degree less than $m$ are in the image of $\phi$. In particular, $\phi(X)=v_{i_{2}} \cdots v_{i_{m}} g$ for some $X \in\left(T(V) \#_{\alpha} G\right)[\hbar]$. Then

$$
\begin{aligned}
\phi\left(v_{i_{1}} X\right) & =\mu\left(v_{i_{1}}, \phi(X)\right) \\
& =\mu\left(v_{i_{1}}, v_{i_{2}} \cdots v_{i_{m}} t_{g}\right) \\
& =v_{i_{1}} \cdots v_{i_{m}} t_{g}+\mu_{1}\left(v_{i_{1}}, v_{i_{2}} \cdots v_{i_{m}} t_{g}\right) \hbar+\mu_{2}\left(v_{i_{1}}, v_{i_{2}} \cdots v_{i_{m}} t_{g}\right) \hbar^{2}+\cdots .
\end{aligned}
$$

Since $\operatorname{deg}\left(\mu_{k}\right)=-2 k$, by the induction hypothesis, each $\mu_{k}\left(v_{i_{1}}, v_{i_{2}} \cdots v_{i_{m}} t_{g}\right)$ is in the image of $\phi$. Therefore, $v_{i_{1}} \cdots v_{i_{m}} t_{g}$ is in the image of $\phi$, and it follows that $\phi$ is surjective.

Finally, we determine the kernel of $\phi$. Since $\operatorname{deg}\left(\mu_{1}\right)=-2$, we can define a bilinear map $\kappa: V \times V \rightarrow \mathbb{C}^{\alpha} G$ by setting $\kappa\left(v_{i}, v_{j}\right):=\mu_{1}\left(v_{i}, v_{j}\right)-q_{i j} \mu_{1}\left(v_{j}, v_{i}\right)$ for all $i, j$. Let $I$ denote the ideal in $\left(T(V) \#_{\alpha} G\right)[\hbar]$ generated by the elements

$$
v_{i} v_{j}-q_{i j} v_{j} v_{i}-\kappa\left(v_{i}, v_{j}\right) \hbar .
$$

Since $\mu_{k}\left(v_{i}, v_{j}\right)=0$ for all $k \geq 2$, we have

$$
\begin{aligned}
& \phi\left(v_{i} v_{j}\right)=\mu\left(v_{i}, v_{j}\right)=v_{i} v_{j}+\mu_{1}\left(v_{i}, v_{j}\right) \hbar, \\
& \phi\left(v_{j} v_{i}\right)=\mu\left(v_{j}, v_{i}\right)=v_{j} v_{i}+\mu_{1}\left(v_{j}, v_{i}\right) \hbar,
\end{aligned}
$$

and so $I$ is contained in the kernel of $\phi$. The form of the relations and surjectivity of $\phi$ imply that the kernel of $\phi$ is precisely $I$, and it follows that the deformation $\left(\left(S_{q}(V) \#_{\alpha} G\right)[\hbar], \mu\right)$ is isomorphic to the twisted quantum Drinfeld Hecke algebra $\mathcal{H}_{\boldsymbol{q}, \kappa, \alpha, \hbar}$ over $\mathbb{C}[\hbar]$.

\section{Computing $\mathrm{HH}^{2}\left(S_{q}(V) \#_{\alpha} G\right)$}

Let $A$ be an algebra on which the finite group $G$ acts by automorphisms, and let $\alpha$ be a 2-cocycle on $G$. This section is concerned with the computation of the Hochschild cohomology $\mathrm{HH}^{*}\left(A \#_{\alpha} G\right)$ of the twisted skew group algebra $A \#_{\alpha} G$. We are particularly interested in degree two cohomology in the case when $A$ is the quantum symmetric algebra $S_{q}(V)$. The results of this section are used in the sections that follow.

Recall that the Hochschild cohomology of an algebra $R$ is

$$
\mathrm{HH}^{*}(R):=\operatorname{Ext}_{R^{e}}(R, R),
$$


where the enveloping algebra $R^{e}:=R \otimes R^{\mathrm{op}}$ acts on $R$ by left and right multiplication. When $R$ is a twisted skew group algebra $A \#_{\alpha} G$ in a characteristic not dividing the order of the finite group $G$, by [Ştefan 1995, Corollary 3.4], there is an action of $G$ on $\mathrm{HH}^{*}\left(A, A \#_{\alpha} G\right)=\operatorname{Ext}_{A^{e}}\left(A, A \#_{\alpha} G\right)$ for which $\mathrm{HH}^{*}\left(A \#_{\alpha} G\right)$ is isomorphic to $\mathrm{HH}^{\circ}\left(A, A \#_{\alpha} G\right)^{G}$, the space of elements of $\mathrm{HH}^{*}\left(A, A \#_{\alpha} G\right)$ that are invariant under the action of $G$. Thus, one can compute $\operatorname{HH}^{\circ}\left(A \#_{\alpha} G\right)$ by first computing $\mathrm{HH}^{\circ}\left(A, A \#_{\alpha} G\right)$ and then determining the space of $G$-invariant elements. When $A$ is the quantum symmetric algebra $S_{q}(V)$, we compute $\mathrm{HH}^{\cdot}\left(S_{q}(V), S_{q}(V) \#_{\alpha} G\right)$ using the quantum Koszul resolution, recalled below.

The quantum exterior algebra $\bigwedge_{\boldsymbol{q}}(V)$ associated to the tuple $\boldsymbol{q}=\left(q_{i j}\right)$ is

$$
\left.\bigwedge_{q}(V):=\mathbb{C}\left\langle v_{1}, \ldots, v_{n}\right| v_{i} v_{j}=-q_{i j} v_{j} v_{i} \text { for all } 1 \leq i, j \leq n\right\rangle .
$$

Since we are working in characteristic 0 , the defining relations imply in particular that $v_{i}^{2}=0$ for each $v_{i}$ in $\bigwedge_{q}(V)$. This algebra has a basis given by all $v_{i_{1}} \cdots v_{i_{m}}$ $\left(0 \leq m \leq n, 1 \leq i_{1}<\cdots<i_{m} \leq n\right)$; we write such a basis element as $v_{i_{1}} \wedge \cdots \wedge v_{i_{m}}$ by analogy with the ordinary exterior algebra.

By [Wambst 1993, Proposition 4.1(c)], the following is a free $S_{q}(V)^{e}$-resolution of $S_{q}(V)$ :

$$
\cdots \rightarrow S_{\boldsymbol{q}}(V)^{e} \otimes \bigwedge_{\boldsymbol{q}}^{2}(V) \stackrel{d_{2}}{\rightarrow} S_{\boldsymbol{q}}(V)^{e} \otimes \bigwedge_{\boldsymbol{q}}^{1}(V) \stackrel{d_{1}}{\rightarrow} S_{\boldsymbol{q}}(V)^{e} \stackrel{\text { mult }}{\rightarrow} S_{\boldsymbol{q}}(V) \rightarrow 0,
$$

that is, for $1 \leq m \leq n$, the degree $m$ term is $S_{\boldsymbol{q}}(V)^{e} \otimes \bigwedge_{\boldsymbol{q}}^{m}(V)$; the differential $d_{m}$ is defined by

$$
\begin{aligned}
& d_{m}\left(1^{\otimes 2} \otimes v_{j_{1}} \wedge \cdots \wedge v_{j_{m}}\right) \\
& =\sum_{i=1}^{m}(-1)^{i+1}\left[\left(\prod_{s=1}^{i} q_{j_{s}, j_{i}}\right) v_{j_{i}} \otimes 1-\left(\prod_{s=i}^{m} q_{j_{i}, j_{s}}\right) \otimes v_{j_{i}}\right] \otimes v_{j_{1}} \wedge \cdots \wedge \hat{v}_{j_{i}} \wedge \cdots \wedge v_{j_{m}}
\end{aligned}
$$

whenever $1 \leq j_{1}<\cdots<j_{m} \leq n$, and mult denotes the multiplication map. The complex (4.1) is a quantum version of the usual Koszul resolution for a polynomial ring.

Suppose that the action of $G$ on $V$ induces an action on $\bigwedge_{q}(V)$. Thus, there is an action of $G$ on the quantum Koszul complex (4.1), that is, an action of $G$ on each $S_{q}(V)^{e} \otimes \bigwedge_{q}^{i}(V)$ that commutes with the differentials.

Assume that $\mathrm{HH}^{\circ}\left(S_{q}(V) \#_{\alpha} G\right)$ has been computed using the quantum Koszul resolution. So, elements of $\mathrm{HH}^{*}\left(S_{\boldsymbol{q}}(V) \#_{\alpha} G\right)$ are given as $G$-invariant elements of $\mathrm{HH}^{*}\left(S_{\boldsymbol{q}}(V), S_{\boldsymbol{q}}(V) \#_{\alpha} G\right)$. For our purposes, we need to find representatives for elements in $\mathrm{HH}^{2}\left(S_{\boldsymbol{q}}(V) \#_{\alpha} G\right)$ that are given as maps from $\left(S_{\boldsymbol{q}}(V) \#_{\alpha} G\right) \otimes\left(S_{\boldsymbol{q}}(V) \#_{\alpha} G\right)$ to $S_{q}(V) \#_{\alpha} G$ satisfying the 2-cocycle condition (3.1). To this end, we consider 
chain maps between the quantum Koszul resolution (4.1) and the bar resolution of $A$ :

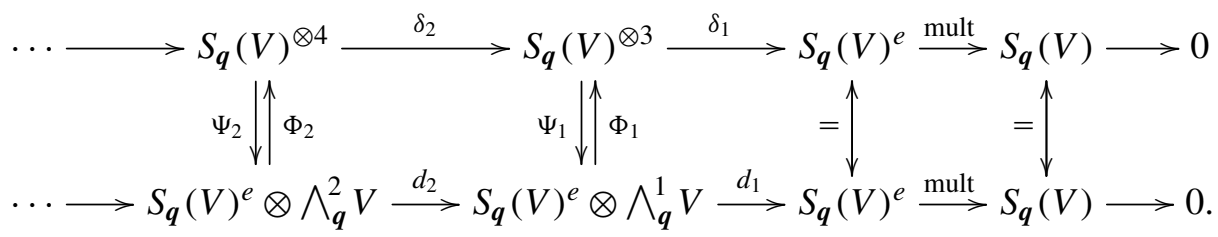

Here the differentials $\delta_{i}$ in the bar resolution are defined as

$$
\delta_{i}\left(a_{0} \otimes \cdots \otimes a_{i+1}\right)=\sum_{j=0}^{i}(-1)^{j} a_{0} \otimes \cdots \otimes a_{j} a_{j+1} \otimes \cdots \otimes a_{i+1}
$$

for all $a_{0}, \ldots, a_{i+1} \in A$. We will only need to know the values of $\Psi_{2}$ on elements of the form $1 \otimes v_{i} \otimes v_{j} \otimes 1$, and these can be chosen to be

$$
\Psi_{2}\left(1 \otimes v_{i} \otimes v_{j} \otimes 1\right)= \begin{cases}1 \otimes 1 \otimes v_{i} \wedge v_{j} & \text { if } i<j, \\ 0 & \text { if } i \geq j .\end{cases}
$$

Chain maps $\Phi_{i}$ are defined in [Naidu et al. 2011], and more generally in [Wambst 1993], that embed the quantum Koszul resolution as a subcomplex of the bar resolution. We will only need $\Phi_{2}$, and this is defined by

$$
\Phi_{m}\left(1 \otimes 1 \otimes v_{i} \wedge \wedge v_{j}\right)=1 \otimes v_{i} \otimes v_{j} \otimes 1-q_{i j} \otimes v_{j} \otimes v_{i} \otimes 1
$$

for all $1 \leq i, j \leq n$.

We define the Reynold's operator, or averaging map, which ensures $G$-invariance of the image, compensating for the possibility that $\Psi_{2}$ may not preserve the action of $G$ :

$$
\begin{aligned}
\mathscr{R}_{2}: \operatorname{Hom}_{\mathbb{C}}\left(S_{\boldsymbol{q}}(V)^{\otimes 2}, S_{\boldsymbol{q}}(V) \#_{\alpha} G\right) & \rightarrow \operatorname{Hom}_{\mathbb{C}}\left(S_{\boldsymbol{q}}(V)^{\otimes 2}, S_{\boldsymbol{q}}(V) \#_{\alpha} G\right)^{G}, \\
\mathscr{R}_{2}(\gamma) & :=\frac{1}{|G|} \sum_{g \in G}{ }^{g} \gamma .
\end{aligned}
$$

The following map tells how to extend a function defined on $S_{q}(V)^{\otimes 2}$ to a function defined on $\left(S_{\boldsymbol{q}}(V) \#_{\alpha} G\right)^{\otimes 2}$ [Căldăraru et al. 2004]:

$$
\begin{gathered}
\Theta_{2}^{*}: \operatorname{Hom}_{\mathbb{C}}\left(S_{\boldsymbol{q}}(V)^{\otimes 2}, S_{\boldsymbol{q}}(V) \#_{\alpha} G\right)^{G} \rightarrow \operatorname{Hom}_{\mathbb{C}}\left(\left(S_{\boldsymbol{q}}(V) \#_{\alpha} G\right)^{\otimes 2}, S_{\boldsymbol{q}}(V) \#_{\alpha} G\right), \\
\Theta_{2}^{*}(\kappa)\left(a_{1} t_{g_{1}} \otimes a_{2} t_{g_{2}}\right):=\alpha\left(g_{1}, g_{2}\right) \kappa\left(a_{1} \otimes{ }^{g_{1}} a_{2}\right) t_{g_{1} g_{2}} .
\end{gathered}
$$

The theorem below is from [Căldăraru et al. 2004]; see also [Shepler and Witherspoon 2012].

Theorem 4.4. Suppose that the action of $G$ on $V$ extends to an action on $\bigwedge_{q}(V)$ by automorphisms. The map

$$
\Theta_{2}^{*} \mathscr{R}_{2} \Psi_{2}^{*}: \operatorname{Hom}_{\mathbb{C}}\left(\bigwedge_{\boldsymbol{q}}^{2}(V), S_{\boldsymbol{q}}(V) \#_{\alpha} G\right) \rightarrow \operatorname{Hom}_{\mathbb{C}}\left(S_{\boldsymbol{q}}(V)^{\otimes 2}, S_{\boldsymbol{q}}(V) \#_{\alpha} G\right)
$$


induces an isomorphism

$$
\mathrm{HH}^{2}\left(S_{\boldsymbol{q}}(V), S_{\boldsymbol{q}}(V) \#_{\alpha} G\right)^{G} \stackrel{\sim}{\rightarrow} \mathrm{HH}^{2}\left(S_{\boldsymbol{q}}(V) \#_{\alpha} G\right) .
$$

Moreover, $\Theta_{2}^{*} \Re_{2} \Psi_{2}^{*}$ maps $\mathrm{HH}^{2}\left(S_{\boldsymbol{q}}(V), S_{\boldsymbol{q}}(V) \#_{\alpha} G\right)$ onto $\mathrm{HH}^{2}\left(S_{\boldsymbol{q}}(V) \#_{\alpha} G\right)$.

Next, we will introduce some notation and give some formulas that are useful in the sections that follow. For each $g \in G$, the space $S_{q}(V) t_{g} \subseteq S_{q}(V) \#_{\alpha} G$ is a (left) $S_{q}(V)^{e}$-module via the action

$$
(a \otimes b) \cdot\left(c t_{g}\right):=a c t_{g} b=a c\left({ }^{g} b\right) t_{g}
$$

for all $a, b, c \in S_{q}(V)$ and all $g \in G$. Note that $\operatorname{HH}^{2}\left(S_{q}(V), S_{q}(V) \#_{\alpha} G\right)$ is isomorphic to the direct sum $\bigoplus_{g \in G} \mathrm{HH}^{2}\left(S_{q}(V), S_{q}(V) t_{g}\right)$.

We wish to express the formula for the differentials $d_{m}$ in the quantum Koszul resolution (4.1) in a more convenient form. To this end, let $\mathbb{N}^{n}$ denote the set of all $n$-tuples of elements from $\mathbb{N}$. The length of $\gamma=\left(\gamma_{1}, \ldots, \gamma_{n}\right) \in \mathbb{N}^{n}$, denoted $|\gamma|$, is the sum $\sum_{i=1}^{n} \gamma_{i}$. For each $\gamma \in \mathbb{N}^{n}$, define $v^{\gamma}:=v_{1}^{\gamma_{1}} v_{2}^{\gamma_{2}} \cdots v_{n}^{\gamma_{n}}$. For each $i \in\{1, \ldots, n\}$, define $[i] \in \mathbb{N}^{n}$ by $[i]_{j}=\delta_{i, j}$, for all $j \in\{1, \ldots, n\}$. For each $\beta=$ $\left(\beta_{1}, \ldots, \beta_{n}\right) \in\{0,1\}^{n}$, let $v^{\wedge \beta}$ denote the vector $v_{j_{1}} \wedge \cdots \wedge v_{j_{m}} \in \bigwedge_{\boldsymbol{q}}^{m}(V)$ determined by the conditions $m=|\beta|, \beta_{j_{k}}=1$ for all $k \in\{1, \ldots, m\}$, and $j_{1}<\ldots<j_{m}$. For each $\beta \in\{0,1\}^{n}$ with $|\beta|=m$, we have

$d_{m}\left(1^{\otimes 2} \otimes v^{\wedge \beta}\right)=\sum_{i=1}^{n} \delta_{\beta_{i}, 1}(-1)^{\sum_{s=1}^{i-1} \beta_{s}}\left[\left(\prod_{s=1}^{i} q_{s, i}^{\beta_{s}}\right) v_{i} \otimes 1-\left(\prod_{s=i}^{n} q_{i, s}^{\beta_{s}}\right) \otimes v_{i}\right] \otimes v^{\wedge(\beta-[i])}$.

Removing the term $S_{q}(V)$ from the quantum Koszul resolution (4.1), applying the functor $\operatorname{Hom}_{S_{q}(V)^{e}}\left(\cdot, S_{q}(V) t_{g}\right)$, and then identifying

$$
\operatorname{Hom}_{S_{q}(V)^{e}}\left(S_{\boldsymbol{q}}(V)^{e} \otimes \bigwedge_{\boldsymbol{q}}^{\bullet}(V), S_{\boldsymbol{q}}(V) t_{g}\right) \cong \operatorname{Hom}_{\mathbb{C}}\left(\bigwedge_{\boldsymbol{q}}^{\bullet}(V), S_{\boldsymbol{q}}(V) t_{g}\right)
$$

with $S_{q}(V) t_{g} \otimes \bigwedge_{q^{-1}}^{\cdot}\left(V^{*}\right)$, we obtain the complex

$$
0 \rightarrow S_{\boldsymbol{q}}(V) t_{g} \stackrel{d_{1}^{*}}{\rightarrow} S_{\boldsymbol{q}}(V) t_{g} \otimes \bigwedge_{q^{-1}}^{1}\left(V^{*}\right) \stackrel{d_{2}^{*}}{\rightarrow} S_{\boldsymbol{q}}(V) t_{g} \otimes \bigwedge_{\boldsymbol{q}^{-1}}^{2}\left(V^{*}\right) \rightarrow \cdots
$$

For all $a \in S_{\boldsymbol{q}}(V)$ and all $\beta \in\{0,1\}^{n}$ with $|\beta|=m-1$, the differential $d_{m}^{*}$ sends the element $a t_{g} \otimes\left(v^{*}\right)^{\wedge \beta}$ to

$$
\sum_{i=1}^{n} \delta_{\beta_{i}, 0}(-1)^{\sum_{s=1}^{i} \beta_{s}}\left[\left(\left(\prod_{s=1}^{i} q_{s, i}^{\beta_{s}}\right) v_{i} a-\left(\prod_{s=i}^{n} q_{i, s}^{\beta_{s}}\right) a\left({ }^{g} v_{i}\right)\right) t_{g}\right] \otimes\left(v^{*}\right)^{\wedge(\beta+[i])} .
$$

For later use, we record the following formula. Let $\eta \in\left(S_{q}(V) \#_{\alpha} G\right) \otimes \bigwedge_{q^{-1}}^{2}\left(V^{*}\right)$. Then

$$
\left[\Theta_{2}^{*} \mathscr{R}_{2} \Psi_{2}^{*}(\eta)\right]\left(v_{i} \otimes v_{j}\right)=\frac{1}{|G|} \sum_{g \in G} g\left(\eta\left(\Psi_{2}\left(1 \otimes^{g^{-1}} v_{i} \otimes^{g^{-1}} v_{j} \otimes 1\right)\right)\right)
$$


The elements of $\left(\left(S_{\boldsymbol{q}}(V) \#_{\alpha} G\right) \otimes \bigwedge_{\boldsymbol{q}^{-1}}^{2}\left(V^{*}\right)\right)^{G}$ that correspond to constant Hochschild 2-cocycles, i.e., those of degree -2 as maps from $\left(S_{q}(V) \#_{\alpha} G\right) \otimes\left(S_{q}(V) \#_{\alpha} G\right)$ to $S_{\boldsymbol{q}}(V) \#_{\alpha} G$, are precisely those in $\left(\mathbb{C}^{\alpha} G \otimes \wedge_{\boldsymbol{q}^{-1}}^{2}\left(V^{*}\right)\right)^{G}$, due to the form of the chain map $\Psi_{2}$. Note that the intersection of the image of $d_{2}^{*}$ with $\mathbb{C}^{\alpha} G \otimes \bigwedge_{q^{-1}}^{2}\left(V^{*}\right)$ is 0 . Applying our earlier formula, letting $\beta=[j]+[k]$,

$$
\begin{aligned}
& d_{3}^{*}\left(t_{g} \otimes v_{j}^{*} \wedge v_{k}^{*}\right) \\
& \quad=\sum_{i \notin\{j, k\}}(-1)^{\sum_{s=1}^{i} \beta_{s}}\left[\left(\left(\prod_{s=1}^{i} q_{s, i}^{\beta_{s}}\right) v_{i}-\left(\prod_{s=i}^{n} q_{i, s}^{\beta_{s}}\right)^{g} v_{i}\right) t_{g}\right] \otimes\left(v^{*}\right)^{\wedge(\beta+[i])} .
\end{aligned}
$$

\section{Constant Hochschild 2-cocycles}

In this section, we establish the following bijection:

$$
\left\{\begin{array}{c}
\text { constant Hochschild } \\
\text { 2-cocycles on } S_{\boldsymbol{q}}(V) \#_{\alpha} G
\end{array}\right\} \longleftrightarrow\left\{\begin{array}{c}
\text { twisted quantum Drinfeld } \\
\text { Hecke algebras } \mathscr{H}_{\boldsymbol{q}, \kappa, \alpha}
\end{array}\right\} .
$$

We also show that every constant Hochschild 2-cocycles on $S_{q}(V) \#_{\alpha} G$ lifts to a deformation of $S_{q}(V) \#_{\alpha} G$.

We use the following two lemmas shortly.

Lemma 5.1. The action of $G$ on $V$ extends to an action on $\bigwedge_{q}(V)$ by automorphisms if, and only if, for all $g \in G, i \neq j$, and $k<l$,

$$
\left(1-q_{i j} q_{l k}\right) g_{k}^{i} g_{l}^{j}+\left(q_{i j}-q_{l k}\right) g_{l}^{i} g_{k}^{j}=0 .
$$

Proof. See [Naidu and Witherspoon 2011, Lemma 4.2].

Lemma 5.2. Suppose that the action of $G$ on $V$ extends to an action, by automorphisms, on $S_{\boldsymbol{q}}(V)$ and on $\bigwedge_{\boldsymbol{q}}(V)$. Then, for all $g \in G$ and all $i, j, k, l(i<j, k<l)$, if $g_{l}^{i} g_{k}^{j} \neq 0$, then $q_{l k}=q_{i j}$, and if $g_{k}^{i} g_{l}^{j} \neq 0$, then $q_{l k}=q_{i j}^{-1}$.

Proof. See [Naidu and Witherspoon 2011, Lemma 4.3].

Proposition 3.5 showed that every twisted quantum Drinfeld Hecke algebra arises from the quantum skew-symmetrization of a constant Hochschild 2-cocycle. The following theorem shows that the converse is also true. The proof of the following theorem involves the maps $\Theta_{2}^{*}, \mathscr{R}_{2}, \Psi_{2}^{*}$, and $d_{3}^{*}$ defined in Section 4.

Theorem 5.3. Suppose that the action of $G$ on $V$ extends to an action, by automorphisms, on $S_{q}(V)$ and on $\bigwedge_{q}(V)$. Let $\alpha$ be a normalized 2-cocycle on $G$, let $\mu_{1}$ be a constant Hochschild 2-cocycle on $S_{q}(V) \#_{\alpha} G$, and let $\kappa: V \times V \rightarrow \mathbb{C}^{\alpha} G$ be the quantum skew-symmetrization of $\mu_{1}$. Then $\mathcal{H}_{\boldsymbol{q}, \kappa, \alpha}$ is a twisted quantum Drinfeld Hecke algebra. 
Proof. We show that the map $\kappa$ satisfies the conditions of Theorem 2.2. Let $\eta$ be a $G$-invariant element of

$$
\operatorname{Hom}_{\mathbb{C}}\left(\bigwedge_{\boldsymbol{q}}^{2}(V), S_{\boldsymbol{q}}(V) \#_{\alpha} G\right) \cong\left(S_{\boldsymbol{q}}(V) \#_{\alpha} G\right) \otimes \bigwedge_{\boldsymbol{q}^{-1}}^{2}\left(V^{*}\right)
$$

such that $\left[\Theta_{2}^{*} \mathscr{R}_{2} \Psi_{2}^{*}\right](\eta)=\mu_{1}$. Since $\mu_{1}$ is a constant Hochschild 2-cocycle, the image of $\eta$ as a map from $\bigwedge_{q}^{2}(V)$ to $S_{q}(V) \#_{\alpha} G$ is contained in $\mathbb{C}^{\alpha} G$, or, equivalently, $\eta$ belongs to $\left(\mathbb{C}^{\alpha} G\right) \otimes \bigwedge_{q^{-1}}^{2}\left(V^{*}\right)$.

For all $1 \leq k, l \leq n$, we have $\left[\Psi_{2}^{*}(\eta)\right]\left(v_{k} \otimes v_{l}-q_{k l} v_{l} \otimes v_{k}\right)=\eta\left(v_{k} \wedge v_{l}\right)$. This equality and the $G$-invariance of $\eta$ imply that $\kappa\left(v_{i}, v_{j}\right)=\eta\left(v_{i} \wedge v_{j}\right)$ for all $1 \leq i, j \leq n$. Indeed, we have

$$
\begin{aligned}
\kappa\left(v_{i}, v_{j}\right) & =\left[\Theta_{2}^{*} \mathscr{R}_{2} \Psi_{2}^{*}(\eta)\right]\left(v_{i} \otimes v_{j}-q_{i j} v_{j} \otimes v_{i}\right) \\
& =\frac{1}{|G|} \sum_{g \in G} \Theta_{2}^{*}\left({ }^{g}\left(\Psi_{2}^{*}(\eta)\right)\right)\left(v_{i} \otimes v_{j}-q_{i j} v_{j} \otimes v_{i}\right) \\
& =\frac{1}{|G|} \sum_{g \in G}^{g}\left(\left(\Psi_{2}^{*}(\eta)\right)^{g^{-1}}\left(v_{i} \otimes v_{j}-q_{i j} v_{j} \otimes v_{i}\right)\right) \\
& =\frac{1}{|G|} \sum_{g \in G} g\left(\left(\Psi_{2}^{*}(\eta)\right)\left(\sum_{k, l}\left(g^{-1}\right)_{k}^{i}\left(g^{-1}\right)_{l}^{j}\left(v_{k} \otimes v_{l}-q_{i j} v_{l} \otimes v_{k}\right)\right)\right) \\
& =\frac{1}{|G|} \sum_{g \in G} g\left(\sum_{k, l}\left(g^{-1}\right)_{k}^{i}\left(g^{-1}\right)_{l}^{j}\left(\Psi_{2}^{*}(\eta)\right)\left(v_{k} \otimes v_{l}-q_{i j} v_{l} \otimes v_{k}\right)\right) \\
& =\frac{1}{|G|} \sum_{g \in G}^{g}\left(\sum_{k, l}\left(g^{-1}\right)_{k}^{i}\left(g^{-1}\right)_{l}^{j} \eta\left(v_{k} \wedge v_{l}\right)\right) \\
& =\frac{1}{|G|} \sum_{g \in G}^{g}\left(\eta\left({ }^{-1}\left(v_{i} \wedge v_{j}\right)\right)\right) \\
& =\frac{1}{|G|} \sum_{g \in G}\left({ }^{g} \eta\right)\left(v_{i} \wedge v_{j}\right) \\
& =\eta\left(v_{i} \wedge v_{j}\right) .
\end{aligned}
$$

Next, write

$$
\eta=\sum_{g \in G} \sum_{1 \leq r<s \leq n} \eta_{r s}^{g} t_{g} \otimes v_{r}^{*} \wedge v_{s}^{*} \in \mathbb{C}^{\alpha} G \otimes \bigwedge_{\boldsymbol{q}^{-1}}^{2}\left(V^{*}\right) \subseteq\left(S_{\boldsymbol{q}}(V) \#_{\alpha} G\right) \otimes \bigwedge_{\boldsymbol{q}^{-1}}^{2}\left(V^{*}\right) .
$$

The calculation above implies that $\kappa_{g}\left(v_{i}, v_{j}\right)=\eta_{i j}^{g}$ for all $i<j$ and all $g \in G$. Since $\eta$ is a Hochschild 2-cocycle, we have $d_{3}^{*}(\eta)=0$. Using (4.8), we see that, for all $1 \leq i<j<k \leq n$, we must have

$\sum_{g \in G}\left(\eta_{j k}^{g} v_{i} t_{g}-\eta_{j k}^{g} q_{i j} q_{i k}^{g} v_{i} t_{g}-\eta_{i k}^{g} q_{i j} v_{j} t_{g}+\eta_{i k}^{g} q_{j k}^{g} v_{j} t_{g}+\eta_{i j}^{g} q_{i k} q_{j k} v_{k} t_{g}-\eta_{i j}^{g} v_{k} t_{g}\right)=0$. 
Equivalently,

$$
-\eta_{j k}^{g}\left(q_{i j} q_{i k}^{g} v_{i}-v_{i}\right)-\eta_{i k}^{g}\left(q_{i j} v_{j}-q_{j k}{ }^{g} v_{j}\right)-\eta_{i j}^{g}\left({ }^{g} v_{k}-q_{i k} q_{j k} v_{k}\right)=0
$$

for all $1 \leq i<j<k \leq n$ and all $g \in G$.

Multiplying both sides by $q_{j i} q_{k i} q_{k j}$ yields

$$
-q_{j k} \eta_{j k}^{g}\left({ }^{g} v_{i}-q_{j i} q_{k i} v_{i}\right)-q_{k i} \eta_{i k}^{g}\left(q_{k j} v_{j}-q_{j i}{ }^{g} v_{j}\right)-q_{j i} \eta_{i j}^{g}\left(q_{k j} q_{k i}{ }^{g} v_{k}-v_{k}\right)=0 .
$$

Now substituting $\kappa_{g}\left(v_{k}, v_{j}\right), \kappa_{g}\left(v_{k}, v_{i}\right)$, and $\kappa_{g}\left(v_{j}, v_{i}\right)$ for $-q_{j k} \eta_{j k}^{g},-q_{k i} \eta_{i k}^{g}$, and $-q_{j i} \eta_{i j}^{g}$, respectively, we obtain

$\kappa_{g}\left(v_{k}, v_{j}\right)\left({ }^{g} v_{i}-q_{j i} q_{k i} v_{i}\right)+\kappa_{g}\left(v_{k}, v_{i}\right)\left(q_{k j} v_{j}-q_{j i}{ }^{g} v_{j}\right)+\kappa_{g}\left(v_{j}, v_{i}\right)\left(q_{k j} q_{k i}{ }^{g} v_{k}-v_{k}\right)=0$,

which is condition (2) of Theorem 2.2.

Next, we show that $\kappa$ also satisfies condition (1) of Theorem 2.2. Since $\eta$ is $G$-invariant, we have $\eta\left({ }^{h} v_{i} \wedge{ }^{h} v_{j}\right)={ }^{h}\left(\eta\left(v_{i} \wedge v_{j}\right)\right)$ for all $i, j$ and all $h \in G$. We have

$$
\begin{aligned}
\eta\left({ }^{h} v_{i} \wedge{ }^{h} v_{j}\right) & =\sum_{k, l} h_{k}^{i} h_{l}^{j} \eta\left(v_{k} \wedge v_{l}\right) \\
& =\sum_{k<l} h_{k}^{i} h_{l}^{j} \eta\left(v_{k} \wedge v_{l}\right)-\sum_{k<l} q_{l k} h_{l}^{i} h_{k}^{j} \eta\left(v_{k} \wedge v_{l}\right) \\
& =\sum_{k<l, g \in G}\left(h_{k}^{i} h_{l}^{j}-q_{l k} h_{l}^{i} h_{k}^{j}\right) \eta_{k l}^{g} t_{g},
\end{aligned}
$$

and for all $i<j$, we have

$$
\begin{aligned}
{ }^{h}\left(\eta\left(v_{i} \wedge v_{j}\right)\right) & =h\left(\sum_{g \in G} \eta_{i j}^{g} t_{g}\right) \\
& =\sum_{g \in G} \eta_{i j}^{g} t_{h} t_{g}\left(t_{h}\right)^{-1} \\
& =\sum_{g \in G} \frac{\alpha(h, g) \alpha\left(h g, h^{-1}\right)}{\alpha\left(h^{-1}, h\right)} \eta_{i j}^{g} t_{h g h^{-1}} \\
& =\sum_{g \in G} \frac{\alpha(h, g)}{\alpha\left(h g h^{-1}, h\right)} \eta_{i j}^{g} t_{h g h^{-1}} .
\end{aligned}
$$

Equating the coefficients of $t_{h g h^{-1}}$, we find that, for all $i<j$ and all $h, g \in G$, we have

$$
\frac{\alpha(h, g)}{\alpha\left(h g h^{-1}, h\right)} \eta_{i j}^{g}=\sum_{k<l}\left(h_{k}^{i} h_{l}^{j}-q_{l k} h_{l}^{i} h_{k}^{j}\right) \eta_{k l}^{h g h^{-1}} .
$$


Substituting $\kappa_{g}\left(v_{i}, v_{j}\right)$ and $\kappa_{h g h^{-1}}\left(v_{k}, v_{l}\right)$ for $\eta_{i j}^{g}$ and $\eta_{k l}^{h g h^{-1}}$, respectively, and then multiplying both sides by $-q_{j i}$ yields

$$
\frac{\alpha(h, g)}{\alpha\left(h g h^{-1}, h\right)} \kappa_{g}\left(v_{j}, v_{i}\right)=\sum_{k<l}\left(q_{j i} q_{l k} h_{l}^{i} h_{k}^{j}-q_{j i} h_{k}^{i} h_{l}^{j}\right) \kappa_{h g h^{-1}}\left(v_{k}, v_{l}\right) .
$$

Substituting $-q_{k l} \kappa_{h g h^{-1}}\left(v_{l}, v_{k}\right)$ for $\kappa_{h g h^{-1}}\left(v_{k}, v_{l}\right)$, and then using Lemma 5.2, we obtain

$$
\frac{\alpha(h, g)}{\alpha\left(h g h^{-1}, h\right)} \kappa_{g}\left(v_{j}, v_{i}\right)=\sum_{k<l} \operatorname{det}_{i j k l}(h) \kappa_{h g h^{-1}}\left(v_{l}, v_{k}\right),
$$

which is condition (1) of Theorem 2.2.

The proof of the following theorem involves the map $\Phi_{2}^{*}$ defined in Section 4.

Theorem 5.4. Let $\alpha$ be a normalized 2-cocycle on $G$. Suppose that the action of $G$ on $V$ extends to an action, by automorphisms, on $S_{q}(V)$ and on $\bigwedge_{q}(V)$. The assignment

$$
\mu_{1} \mapsto \mathscr{H}_{\boldsymbol{q}, \kappa, \alpha}
$$

where $\kappa$ is the quantum skew-symmetrization of $\mu_{1}$ is a bijection from the space of equivalence classes of constant Hochschild 2-cocycles on $S_{q}(V) \#_{\alpha} G$ to the space of twisted quantum Drinfeld Hecke algebras associated to the quadruple $(G, V, \boldsymbol{q}, \alpha)$. Proof. Proposition 3.5 showed that the assignment specified in the statement of the theorem is surjective. To see that the assignment is also injective, let $\mu_{1}$ and $\mu_{1}^{\prime}$ be constant Hochschild 2-cocycles on $S_{q}(V) \#_{\alpha} G$ such that their quantum skewsymmetrizations are equal. We have

$$
\begin{aligned}
{\left[\Phi_{2}^{*}\left(\mu_{1}\right)\right]\left(1 \otimes 1 \otimes v_{i} \otimes v_{j}\right) } & =\mu_{1}\left(v_{i}, v_{j}\right)-q_{i j} \mu_{1}\left(v_{j}, v_{i}\right) \\
& =\mu_{1}^{\prime}\left(v_{i}, v_{j}\right)-q_{i j} \mu_{1}^{\prime}\left(v_{j}, v_{i}\right) \\
& =\left[\Phi_{2}^{*}\left(\mu_{1}^{\prime}\right)\right]\left(1 \otimes 1 \otimes v_{i} \otimes v_{j}\right),
\end{aligned}
$$

so $\Phi_{2}^{*}\left(\mu_{1}\right)=\Phi_{2}^{*}\left(\mu_{1}^{\prime}\right)$, and it follows that $\mu_{1}$ and $\mu_{1}^{\prime}$ are cohomologous.

Theorem 5.5. Let $\alpha$ be a normalized 2-cocycle on G. Suppose that the action of $G$ on $V$ extends to an action, by automorphisms, on $S_{q}(V)$ and on $\bigwedge_{q}(V)$. Each constant Hochschild 2-cocycle on $S_{q}(V) \#_{\alpha} G$ lifts to a deformation of $S_{q}(V) \#_{\alpha} G$ over $\mathbb{C}[\hbar]$.

Proof. Let $\mu_{1}^{\prime}$ be a constant Hochschild 2-cocycle on $S_{q}(V) \#_{\alpha} G$. By Theorem 5.3, $\mu_{1}^{\prime}$ gives rise to a twisted quantum Drinfeld Hecke algebra $\mathscr{H}_{\boldsymbol{q}, \kappa, \alpha}$, where $\kappa$ is the quantum skew-symmetrization of $\mu_{1}^{\prime}$. By Lemma 3.3, $\mathscr{H}_{\boldsymbol{q}, \kappa, \alpha, \hbar}$ is a twisted quantum Drinfeld Hecke algebra over $\mathbb{C}[\hbar]$. By Theorem 3.2, associated to $\mathscr{H}_{\boldsymbol{q}, \kappa, \alpha, \hbar}$ is a deformation $\mu=\mu_{0}+\mu_{1} \hbar+\mu_{2} \hbar^{2}+\cdots$ of $S_{q}(V) \#_{\alpha} G$. The proof of Proposition 3.5 shows that $\kappa$ is the quantum skew-symmetrization of $\mu_{1}$, and it follows from Theorem 5.4 that $\mu_{1}^{\prime}$ is cohomologous to $\mu_{1}$. 


\section{Diagonal actions}

As before, let $G$ be a finite group acting linearly on a vector space $V$ with basis $v_{1}, \ldots, v_{n}$. Assume that $v_{1}, \ldots, v_{n}$ are common eigenvectors for $G$. In this case, the Hochschild cohomology $\mathrm{HH}^{*}\left(S_{q}(V), S_{q}(V) \# G\right)$ was computed in [Naidu et al. 2011]. Let $\alpha$ be a normalized 2-cocycle on $G$. In this section, we use results from [Naidu et al. 2011] to give an explicit description of the subspace of $\mathrm{HH}^{2}\left(S_{\boldsymbol{q}}(V) \#_{\alpha} G\right)$ consisting of constant Hochschild 2-cocycles. As a consequence, we obtain a classification of twisted quantum Drinfeld Hecke algebras associated to the quadruple $(G, V, \boldsymbol{q}, \alpha)$.

Let $\lambda_{g, i} \in \mathbb{C}$ be the scalars for which ${ }^{g} v_{i}=\lambda_{g, i} v_{i}$ for all $g \in G$ and all $i \in\{1, \ldots, n\}$. For each $g \in G$, define

$$
C_{g}:=\left\{\gamma \in(\mathbb{N} \cup\{-1\})^{n} \mid \text { for each } i \in\{1, \ldots, n\}, \prod_{s=1}^{n} q_{i s}^{\gamma_{s}}=\lambda_{g, i} \text { or } \gamma_{i}=-1\right\} .
$$

Theorem 6.2 [Naidu et al. 2011]. If $G$ acts diagonally on $V$, then

$$
\mathrm{HH}^{*}\left(S_{q}(V), S_{q}(V) \# G\right)
$$

is isomorphic to the graded vector subspace of $\left(S_{\boldsymbol{q}}(V) \#_{G}\right) \otimes \bigwedge_{q^{-1}}\left(V^{*}\right)$ given by

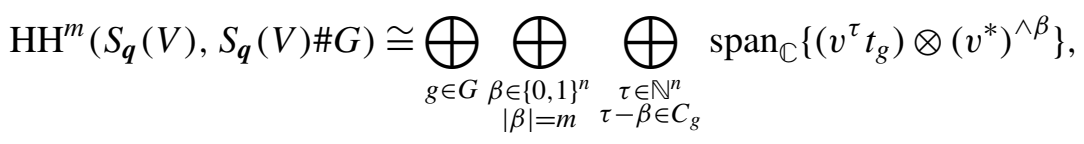

for all $m \in \mathbb{N}$.

Corollary 6.3. The constant Hochschild 2-cocycles representing elements in the cohomology $\mathrm{HH}^{2}\left(S_{q}(V), S_{q}(V) \# G\right)$ form a vector space having as a basis the set of all

$$
t_{g} \otimes v_{r}^{*} \wedge v_{s}^{*},
$$

where $r<s$ and $g \in G$ satisfy $q_{r r^{\prime}} q_{s r^{\prime}}=\lambda_{g, r^{\prime}}$ for all $r^{\prime} \notin\{r, s\}$.

Note that the $S_{q}(V)$-bimodule structure of $S_{q}(V) \#_{\alpha} G$ does not depend on the 2-cocycle $\alpha$, and so $\mathrm{HH}^{2}\left(S_{\boldsymbol{q}}(V), S_{\boldsymbol{q}}(V) \#_{\alpha} G\right)=\mathrm{HH}^{2}\left(S_{\boldsymbol{q}}(V), S_{\boldsymbol{q}}(V) \# G\right)$.

Let $\mathscr{R}$ denote a complete set of representatives of conjugacy classes in $G$, let $C_{G}(a)$ denote the centralizer of $a$ in $G$, and let $\left[G / C_{G}(a)\right]$ denote a complete set of representatives of left cosets of $C_{G}(a)$ in $G$. In the following theorem, the notation $\delta_{i, j}$ is the Kronecker delta.

Theorem 6.4. The constant Hochschild 2-cocycles representing elements in the cohomology $\mathrm{HH}^{2}\left(S_{q}(V) \#_{\alpha} G\right)$ form a vector space having as a basis the set of all

$$
\sum_{g \in\left[G / C_{G}(a)\right]} \frac{\alpha(g, a)}{\alpha\left(g a g^{-1}, g\right)} \lambda_{g, r}^{-1} \lambda_{g, s}^{-1} t_{g a g^{-1}} \otimes v_{r}^{*} \wedge v_{s}^{*},
$$


where $r<s$ and $a \in \mathscr{R}$ satisfy $q_{r r^{\prime}} q_{s r^{\prime}}=\lambda_{a, r^{\prime}}$ for all $r^{\prime} \notin\{r, s\}$, and $\lambda_{h, r} \lambda_{h, s}=$ $\alpha(h, a) / \alpha(a, h)$ for all $h \in C_{G}(a)$.

Proof. We show that the space of $G$-invariant elements of the vector space given in Corollary 6.3 is precisely the vector space stated in the theorem. The stated result then follows from Theorem 4.4.

First, we show that the scalar $\left(\alpha(g, a) / \alpha\left(g_{g} g^{-1}, g\right)\right) \lambda_{g, r}^{-1} \lambda_{g, s}^{-1}$ is independent of choice of representative $g$ of a coset of $C_{G}(a)$ under the assumption that $\lambda_{h, r} \lambda_{h, s}=$ $\alpha(h, a) / \alpha(a, h)$ for all $h \in C_{G}(a)$. Suppose that $g a g^{-1}=g^{\prime} a g^{\prime-1}$. Then $g^{\prime}=g h$ for some $h \in C_{G}(a)$, and we have

$$
\frac{\alpha\left(g^{\prime}, a\right)}{\alpha\left(g^{\prime} a g^{\prime-1}, g^{\prime}\right)} \lambda_{g^{\prime}, r}^{-1} \lambda_{g^{\prime}, s}^{-1}=\frac{\alpha(g h, a)}{\alpha\left(g a g^{-1}, g h\right)} \lambda_{g, r}^{-1} \lambda_{g, s}^{-1} \lambda_{h, r}^{-1} \lambda_{h, s}^{-1} .
$$

Substituting $\lambda_{h, r} \lambda_{h, s}=\alpha(h, a) / \alpha(a, h)$ yields

$$
\frac{\alpha(g h, a) \alpha(a, h)}{\alpha\left(g a g^{-1}, g h\right) \alpha(h, a)} \lambda_{g, r}^{-1} \lambda_{g, s}^{-1} .
$$

Applying the 2-cocycle condition of $\alpha$ to the triple $(g, h, a)$ gives

$$
\alpha(g h, a) / \alpha(h, a)=\alpha(g, h a) / \alpha(g, h) .
$$

Making this substitution in the expression above yields

$$
\frac{\alpha(g, h a) \alpha(a, h)}{\alpha\left(g a g^{-1}, g h\right) \alpha(g, h)} \lambda_{g, r}^{-1} \lambda_{g, s}^{-1} .
$$

Applying the 2-cocycle condition of $\alpha$ to the triple $(g, a, h)$ gives $\alpha(g, h a) \alpha(a, h)=$ $\alpha(g a, h) \alpha(g, a)$. Making this substitution in the expression above yields

$$
\frac{\alpha(g a, h) \alpha(g, a)}{\alpha\left(g a g^{-1}, g h\right) \alpha(g, h)} \lambda_{g, r}^{-1} \lambda_{g, s}^{-1} .
$$

Finally, applying the 2-cocycle condition of $\alpha$ to the triple $\left(\mathrm{gag}^{-1}, g, h\right)$ gives

$$
\frac{\alpha(g a, h)}{\alpha\left(g a g^{-1}, g h\right) \alpha(g, h)}=\frac{1}{\alpha\left(g a g^{-1}, g\right)} \text {. }
$$

Making this substitution in the expression above yields

$$
\frac{\alpha(g, a)}{\alpha\left(g a g^{-1}, g\right)} \lambda_{g, r}^{-1} \lambda_{g, s}^{-1},
$$

proving that the scalar above is independent of choice of representative $g$ of a coset of $C_{G}(a)$ under the assumption that $\lambda_{h, r} \lambda_{h, s}=\alpha(h, a) / \alpha(a, h)$ for all $h \in C_{G}(a)$. Thus, each of the alleged basis element is well defined, and is evidently $G$-invariant. 
Conversely, let $\eta=\sum_{a \in G} \sum \eta_{r s}^{a} t_{a} \otimes v_{r}^{*} \wedge v_{s}^{*}$, where $\eta_{r s}^{a}$ are scalars and the second sum runs over all $r<s$ that satisfy $q_{r r^{\prime}} q_{s r^{\prime}}=\lambda_{a, r^{\prime}}$ for all $r^{\prime} \notin\{r, s\}$. We have ${ }^{g} \eta=\sum_{a \in G} \eta_{r s}^{a} t_{g} t_{a}\left(t_{g}\right)^{-1} \otimes^{g}\left(v_{r}^{*}\right) \wedge^{g}\left(v_{s}^{*}\right)=\sum_{a \in G} \frac{\alpha(g, a)}{\alpha\left(g a g^{-1}, g\right)} \lambda_{g, r}^{-1} \lambda_{g, s}^{-1} \eta_{r s}^{a} t_{g a g^{-1}} \otimes v_{r}^{*} \wedge v_{s}^{*}$. Assume that $\eta$ is $G$-invariant. Then

$$
\eta_{r s}^{g a g^{-1}}=\frac{\alpha(g, a)}{\alpha\left(g a g^{-1}, g\right)} \lambda_{g, r}^{-1} \lambda_{g, s}^{-1} \eta_{r s}^{a},
$$

for all $g \in G$. Letting $g=h \in C_{G}(a)$ yields

$$
\lambda_{h, r} \lambda_{h, s}=\frac{\alpha(h, a)}{\alpha(a, h)},
$$

showing that $\eta$ is in the span of the alleged basis elements. The stated result now follows from Theorem 4.4.

The proof of the following theorem involves the maps $\Theta_{2}^{*}, \mathscr{R}_{2}$, and $\Psi_{2}^{*}$ defined in Section 4.

Theorem 6.5. The maps $\kappa: V \times V \rightarrow \mathbb{C}^{\alpha} G$ for which $\mathscr{H}_{\boldsymbol{q}, \kappa, \alpha}$ is a twisted quantum Drinfeld Hecke algebra form a vector space with basis consisting of maps

$$
\begin{aligned}
f_{r, s, a}: V \times V & \rightarrow \mathbb{C}^{\alpha} G, \\
\left(v_{i}, v_{j}\right) & \mapsto\left(\delta_{i, r} \delta_{j, s}-q_{s r} \delta_{i, s} \delta_{j, r}\right) \sum_{g \in\left[G / C_{G}(a)\right]} \frac{\alpha(g, a)}{\alpha\left(g a g^{-1}, g\right)} \lambda_{g, r}^{-1} \lambda_{g, s}^{-1} t_{g a g^{-1}},
\end{aligned}
$$

where $r<s$ and $a \in \mathscr{R}$ satisfy $q_{r r^{\prime}} q_{s r^{\prime}}=\lambda_{a, r^{\prime}}$ for all $r^{\prime} \notin\{r, s\}$ and $\lambda_{h, r} \lambda_{h, s}=$ $\alpha(h, a) / \alpha(a, h)$ for all $h \in C_{G}(a)$.

Proof. Let $\eta=\sum_{g \in\left[G / C_{G}(a)\right]}\left(\alpha(g, a) / \alpha\left(g a g^{-1}, g\right)\right) \lambda_{g, r}^{-1} \lambda_{g, s}^{-1} t_{g a g^{-1}} \otimes v_{r}^{*} \wedge v_{s}^{*}$, where $r<s$ and $a \in \mathscr{R}$ satisfy the conditions specified in Theorem 6.4. In the proof of Theorem 5.3 we saw that $\left[\Theta_{2}^{*} \mathscr{R}_{2} \Psi_{2}^{*}(\eta)\right]\left(v_{i} \otimes v_{j}-q_{i j} v_{j} \otimes v_{i}\right)=\eta\left(v_{i} \wedge v_{j}\right)$, and the latter is equal to

$$
\left(\delta_{i, r} \delta_{j, s}-q_{s r} \delta_{i, s} \delta_{j, r}\right) \sum_{g \in\left[G / C_{G}(a)\right]} \frac{\alpha(g, a)}{\alpha\left(g_{a g}^{-1}, g\right)} \lambda_{g, r}^{-1} \lambda_{g, s}^{-1} t_{g a g^{-1}} .
$$

The stated result now follows from Theorems 4.4 and 5.4.

\section{Symmetric groups: natural representations}

In this section, we classify twisted quantum Drinfeld Hecke algebras for the symmetric groups $S_{n}, n \geq 4$, acting naturally on a vector space of dimension $n$.

Consider the natural action of $S_{n}$ on a vector space $V$ with ordered basis $v_{1}, \ldots, v_{n}$. Let $\boldsymbol{q}:=\left(q_{i j}\right)_{1 \leq i, j \leq n}$ denote a tuple of nonzero scalars for which $q_{i i}=1$ and $q_{j i}=q_{i j}^{-1}$ for all $i, j$. The action of $S_{n}$ extends to an action on the 
quantum symmetric algebra $S_{q}(V)$ by automorphisms if and only if either $q_{i j}=1$ for all $i, j$ or $q_{i j}=-1$ for all $i \neq j$. The tuple corresponding to the former will be denoted by $\mathbf{1}$, and the tuple corresponding to the latter by $\mathbf{- 1}$. The action of $S_{n}$ on $V$ extends to an action on the quantum exterior algebra $\bigwedge_{-1}$ by automorphisms. Note that the algebra $\bigwedge_{-1}$ is commutative.

The Schur multiplier $\mathrm{H}^{2}\left(S_{n}, \mathbb{C}^{\times}\right)$of the symmetric group $S_{n}$ is isomorphic to $\mathbb{Z} / 2 \mathbb{Z}$ for all $n \geq 4$ [Schur 2001]. Let $\alpha$ be a 2-cocycle on $S_{n}$, and let [ $\alpha$ ] denote the image of $\alpha$ in $\mathrm{H}^{2}\left(S_{n}, \mathbb{C}^{\times}\right)$. A classification of twisted quantum Drinfeld Hecke algebras for $S_{n}$, acting naturally on a vector space of dimension $n$, is given in [Ram and Shepler 2003] for $[\alpha]=1$ and $\boldsymbol{q}=\mathbf{1}$, in [Wambst 1993] for $[\alpha] \neq 1$ and $\boldsymbol{q}=\mathbf{1}$, and in [Naidu and Witherspoon 2011] for $[\alpha]=1$ and $\boldsymbol{q}=\mathbf{- 1}$. The goal of this section is to address the remaining case: $[\alpha] \neq 1$ and $\boldsymbol{q}=-\mathbf{1}$.

Next, we recall a Schur covering group of $S_{n}$, which we use to obtain a cohomologically nontrivial 2-cocycle on $S_{n}$. Let $T_{n}$ be the group with generators $t_{1}, \ldots, t_{n-1}, z$ and relations

$$
\begin{aligned}
z^{2} & =1, & & \\
t_{r}^{2} & =1 & & \text { for } 1 \leq r \leq n-1, \\
t_{r} t_{s} & =t_{s} t_{r} z & & \text { for }|r-s|>1 \text { and } 1 \leq r, s \leq n-1, \\
t_{r} t_{r+1} t_{r} & =t_{r+1} t_{r} t_{r+1} & & \text { for } 1 \leq r \leq n-2, \\
z t_{r} & =t_{r} z & & \text { for } 1 \leq r \leq n-1 .
\end{aligned}
$$

The group $T_{n}$ is a central extension of $S_{n}$ by $\langle z\rangle$ :

$$
1 \rightarrow\langle z\rangle \rightarrow T_{n} \stackrel{p}{\rightarrow} S_{n} \rightarrow 1,
$$

where the surjection $p$ sends $z$ to 1 and sends $t_{r}$ to the transposition $(r r+1)$. The group $T_{n}$ is a Schur covering group of $S_{n}$ [Schur 2001].

We define certain distinguished elements of $T_{n}$ : For every $r, s \in\{1, \ldots, n\}, r \neq s$, denote by $[r s]$ the element of $T_{n}$ defined recursively as follows:

$$
\begin{aligned}
{[r r+1] } & :=t_{r}, & & \\
{[r s] } & :=t_{r}[r+1 s] t_{r} z & & \text { if } r<s-1, \\
{[r s] } & :=[s r] z & & \text { if } r>s .
\end{aligned}
$$

Note that $p([r s])=(r s)$.

Next, we define a section $u: S_{n} \rightarrow T_{n}$ of the surjection $p: T_{n} \rightarrow S_{n}$ by $u(\sigma)=u_{\sigma}$. If $\sigma \in S_{n}$ is the $k$-cycle $\left(a_{1}, \ldots, a_{k}\right)$, where $a_{1}, \ldots, a_{k} \in\{1, \ldots, n\}$ and $a_{1}$ is the smallest element of the set $\left\{a_{1}, \ldots, a_{k}\right\}$, define

$$
u_{\sigma}:=\left[a_{1} a_{k}\right]\left[a_{1} a_{k-1}\right] \cdots\left[a_{1} a_{2}\right] .
$$


If $\sigma \in S_{n}$ is the product $\left(a_{1}, \ldots, a_{k}\right)\left(b_{1}, \ldots, b_{l}\right) \ldots$ of disjoint cycles, where $a_{1}$ is the smallest element of the set $\left\{a_{1}, \ldots, a_{k}\right\}, b_{1}$ is the smallest element of the set $\left\{b_{1}, \ldots, b_{l}\right\}$, and so on, and $a_{1}<b_{1}<\cdots$, define

$$
u_{\sigma}:=u_{\left(a_{1}, \ldots, a_{k}\right)} u_{\left(b_{1}, \ldots, b_{l}\right)} \cdots \text {. }
$$

It is evident that $u: S_{n} \rightarrow T_{n}$ is a section, that is, $p u=\operatorname{id}_{S_{n}}$.

Consider any irreducible representation of the group $T_{n}$. Since the element $z$ is central and has order two, it must necessarily act on this representation as multiplication by either 1 or -1 . Assume the latter. In this case, we obtain a cohomologically nontrivial (normalized) 2-cocyle $\alpha: S_{n} \times S_{n} \rightarrow \mathbb{C}^{\times}$defined by

$$
\alpha(\sigma, \tau):= \begin{cases}1 & \text { if } u_{\sigma} u_{\tau} u_{\sigma \tau}^{-1}=1 \\ -1 & \text { if } u_{\sigma} u_{\tau} u_{\sigma \tau}^{-1}=z\end{cases}
$$

for all $\sigma, \tau \in S_{n}$.

Our goal is to classify twisted quantum Drinfeld Hecke algebras associated to the quadruple $\left(S_{n}, V,-\mathbf{1}, \alpha\right)$, where $V$ is the natural representation of $S_{n}$ and $\mathbf{- 1}$ is the tuple defined earlier in this section. To this end, in what follows, we establish several lemmas that aid in accomplishing our goal.

Since the subgroup $\langle z\rangle$ of $T_{n}$ is central, there is an action of $S_{n}$ on $T_{n}$ induced by conjugation. If $\sigma$ belongs to $S_{n}$ and $v$ belongs to $T_{n}$, we denote by $\sigma \triangleright v$ the result of $\sigma$ acting upon $v$. We have $\sigma \triangleright v=\hat{\sigma} v(\hat{\sigma})^{-1}$, where $\hat{\sigma}$ is any element in the set $p^{-1}(\sigma)$.

For each $\sigma \in S_{n}$, let $\epsilon(\sigma)$ denote the signature of $\sigma$ :

$$
\epsilon(\sigma)= \begin{cases}0 & \text { if } \sigma \text { is an even permutation, } \\ 1 & \text { if } \sigma \text { is an odd permutation. }\end{cases}
$$

The following result from [Vendramin 2012] will be put to use shortly.

Lemma 7.2. For all distinct $r, s \in\{1, \ldots, n\}$ and all $\sigma \in S_{n}$, we have

$$
\sigma \triangleright[r s]=[\sigma(r) \sigma(s)] z^{\epsilon(\sigma)} .
$$

For later use, we record two lemmas.

Lemma 7.3. For all distinct $r, r^{\prime}, s, s^{\prime} \in\{1, \ldots, n\}$, we have

$$
[r s]\left[s r^{\prime}\right] z=\left[r r^{\prime}\right][r s]=\left[r^{\prime} s\right]\left[r r^{\prime}\right] z .
$$

Proof. We have $[r s]^{-1}\left[r r^{\prime}\right][r s]=(r s)^{-1} \triangleright\left[r r^{\prime}\right]=(r s) \triangleright\left[r r^{\prime}\right]$, and, by Lemma 7.2, the last expression equals $\left[s r^{\prime}\right] z$, proving the first equality. The second equality is proved similarly.

Lemma 7.4. For all distinct $r, r^{\prime}, s, s^{\prime} \in\{1, \ldots, n\}$, we have

$$
[r s]\left[r^{\prime} s^{\prime}\right]=\left[r^{\prime} s^{\prime}\right][r s] z .
$$


Proof. We have $[r s]\left[r^{\prime} s^{\prime}\right][r s]^{-1}=(r s) \triangleright\left[r^{\prime} s^{\prime}\right]$, and, by Lemma 7.2, the last expression equals $\left[r^{\prime} s^{\prime}\right] z$.

For all distinct $r, s, r^{\prime}, s^{\prime} \in\{1, \ldots, n\}$, let $d\left(r, s, r^{\prime}, s^{\prime}\right)$ denote the number of inequalities

$$
\min \{r, s\}>\min \left\{r^{\prime}, s^{\prime}\right\}, \quad r>s, \quad r^{\prime}>s^{\prime}
$$

that hold. For all distinct $r, s, r^{\prime}, s^{\prime} \in\{1, \ldots, n\}$ and all $\sigma \in S_{n}$, define

$$
d_{\sigma}\left(r, s, r^{\prime}, s^{\prime}\right):=d\left(\sigma(r), \sigma(s), \sigma\left(r^{\prime}\right), \sigma\left(s^{\prime}\right)\right) .
$$

For later use, we record the following obvious result.

Lemma 7.5. For all distinct $r, s, r^{\prime}, s^{\prime} \in\{1, \ldots, n\}$, we have

$$
\left|d\left(r, s, r^{\prime}, s^{\prime}\right)-d\left(r, s, s^{\prime}, r^{\prime}\right)\right|=1=\left|d\left(r, s, r^{\prime}, s^{\prime}\right)-d\left(s, r, r^{\prime}, s^{\prime}\right)\right| .
$$

We need the following lemma, which is a generalization of [Vendramin 2012, Lemma 3.7].

Lemma 7.6. Let $\sigma$ be any element of $S_{n}$.

(a) For all $r, s \in\{1, \ldots, n\}$ with $r<s$, we have

$$
\sigma \triangleright u_{(r s)}= \begin{cases}u_{\sigma(r s) \sigma^{-1}} z^{\epsilon(\sigma)} & \text { if } \sigma(r)<\sigma(s), \\ u_{\sigma(r s) \sigma^{-1}} z^{\epsilon(\sigma)+1} & \text { if } \sigma(r)>\sigma(s) .\end{cases}
$$

(b) For all distinct $r, s, r^{\prime}, s^{\prime} \in\{1, \ldots, n\}$ with $r<s, r^{\prime}<s^{\prime}$, and $r<r^{\prime}$, we have

$$
\sigma \triangleright u_{(r s)\left(r^{\prime} s^{\prime}\right)}=u_{\sigma(r s)\left(r^{\prime} s^{\prime}\right) \sigma^{-1}} z^{d_{\sigma}\left(r, s, r^{\prime}, s^{\prime}\right)} .
$$

(c) For all distinct $r, s, r^{\prime} \in\{1, \ldots, n\}$ with $r<s$ and $r<r^{\prime}$, we have

$$
\sigma \triangleright u_{\left(r s r^{\prime}\right)}=u_{\sigma\left(r s r^{\prime}\right) \sigma^{-1}}
$$

Proof. (a) By Lemma 7.2, $\sigma \triangleright u_{(r s)}=\sigma \triangleright[r s]=[\sigma(r) \sigma(s)] z^{\epsilon(\sigma)}$. If $\sigma(r)<\sigma(s)$, then

$$
[\sigma(r) \sigma(s)] z^{\epsilon(\sigma)}=u_{(\sigma(r) \sigma(s))} z^{\epsilon(\sigma)}=u_{\sigma(r s) \sigma^{-1}} z^{\epsilon(\sigma)} .
$$

If $\sigma(r)>\sigma(s)$, then

$$
[\sigma(r) \sigma(s)] z^{\epsilon(\sigma)}=[\sigma(s) \sigma(r)] z^{\epsilon(\sigma)+1}=u_{(\sigma(s) \sigma(r))} z^{\epsilon(\sigma)+1}=u_{\sigma(r s) \sigma^{-1}} z^{\epsilon(\sigma)+1} .
$$

(b) Again, by Lemma 7.2,

$$
\begin{aligned}
\sigma \triangleright u_{(r s)\left(r^{\prime} s^{\prime}\right)}=\sigma \triangleright[r s]\left[r^{\prime} s^{\prime}\right] & =(\sigma \triangleright[r s])\left(\sigma \triangleright\left[r^{\prime} s^{\prime}\right]\right) \\
& =[\sigma(r) \sigma(s)] z^{\epsilon(\sigma)}\left[\sigma\left(r^{\prime}\right) \sigma\left(s^{\prime}\right)\right] z^{\epsilon(\sigma)} \\
& =[\sigma(r) \sigma(s)]\left[\sigma\left(r^{\prime}\right) \sigma\left(s^{\prime}\right)\right] .
\end{aligned}
$$

If $\min \{\sigma(r), \sigma(s)\}>\min \left\{\sigma\left(r^{\prime}\right), \sigma\left(s^{\prime}\right)\right\}$, then, using Lemma 7.4, we rewrite the product above as $\left[\sigma\left(r^{\prime}\right) \sigma\left(s^{\prime}\right)\right][\sigma(r) \sigma(s)] z$. If $\sigma(r)>\sigma(s)$, we replace $[\sigma(r) \sigma(s)]$ by 
$[\sigma(s) \sigma(r)] z$. Similarly, if $\sigma\left(r^{\prime}\right)>\sigma\left(s^{\prime}\right)$, we replace $\left[\sigma\left(r^{\prime}\right) \sigma\left(s^{\prime}\right)\right]$ by $\left[\sigma\left(s^{\prime}\right) \sigma\left(r^{\prime}\right)\right] z$. Since the element $z$ has order two, the stated result follows. For example, suppose that $d_{\sigma}\left(r, s, r^{\prime}, s^{\prime}\right)=3$. Then $\sigma(r)>\sigma(s), \sigma\left(r^{\prime}\right)>\sigma\left(s^{\prime}\right)$, and $\sigma(s)>\sigma\left(s^{\prime}\right)$, and in this case we write

$$
\begin{aligned}
{[\sigma(r) \sigma(s)]\left[\sigma\left(r^{\prime}\right) \sigma\left(s^{\prime}\right)\right]=\left[\sigma\left(r^{\prime}\right) \sigma\left(s^{\prime}\right)\right][\sigma(r) \sigma(s)] z } & =\left[\sigma\left(s^{\prime}\right) \sigma\left(r^{\prime}\right)\right] z[\sigma(s) \sigma(r)] z z \\
& =\left[\sigma\left(s^{\prime}\right) \sigma\left(r^{\prime}\right)\right][\sigma(s) \sigma(r)] z \\
& =u_{\left(\sigma\left(s^{\prime}\right) \sigma\left(r^{\prime}\right)\right)(\sigma(s) \sigma(r))} \\
& =u_{\sigma(r s)\left(r^{\prime} s^{\prime}\right) \sigma^{-1} z}
\end{aligned}
$$

(c) Again, by Lemma 7.2,

$$
\begin{aligned}
\sigma \triangleright u_{\left(r s r^{\prime}\right)}=\sigma \triangleright\left[r r^{\prime}\right][r s] & =\left(\sigma \triangleright\left[r r^{\prime}\right]\right)(\sigma \triangleright[r s]) \\
& =\left[\sigma(r) \sigma\left(r^{\prime}\right)\right] z^{\epsilon(\sigma)}[\sigma(r) \sigma(s)] z^{\epsilon(\sigma)} \\
& =\left[\sigma(r) \sigma\left(r^{\prime}\right)\right][\sigma(r) \sigma(s)] .
\end{aligned}
$$

Case $\left(\mathrm{c}_{1}\right) . \sigma(r)<\sigma\left(r^{\prime}\right)$ and $\sigma(r)<\sigma(s)$. In this case,

$$
\left[\sigma(r) \sigma\left(r^{\prime}\right)\right][\sigma(r) \sigma(s)]=u_{\left(\sigma(r) \sigma(s) \sigma\left(r^{\prime}\right)\right)}=u_{\sigma\left(r s r^{\prime}\right) \sigma^{-1}} .
$$

Case $\left(\mathrm{c}_{2}\right)$. Either $\sigma(s)<\sigma(r)<\sigma\left(r^{\prime}\right)$ or $\sigma(s)<\sigma\left(r^{\prime}\right)<\sigma(r)$. Using the first equality of Lemma 7.3,

$$
\begin{aligned}
{\left[\sigma(r) \sigma\left(r^{\prime}\right)\right][\sigma(r) \sigma(s)]=[\sigma(r) \sigma(s)]\left[\sigma(s) \sigma\left(r^{\prime}\right)\right] z } & =[\sigma(s) \sigma(r)] z\left[\sigma(s) \sigma\left(r^{\prime}\right)\right] z \\
& =u_{\left(\sigma(s) \sigma\left(r^{\prime}\right) \sigma(r)\right)} \\
& =u_{\sigma\left(r s r^{\prime}\right) \sigma^{-1}}
\end{aligned}
$$

Case ( $\left.\mathrm{c}_{3}\right)$. Either $\sigma\left(r^{\prime}\right)<\sigma(r)<\sigma(s)$ or $\sigma\left(r^{\prime}\right)<\sigma(s)<\sigma(r)$. Using the second equality of Lemma 7.3,

$$
\begin{aligned}
{\left[\sigma(r) \sigma\left(r^{\prime}\right)\right][\sigma(r) \sigma(s)] } & =\left[\sigma\left(r^{\prime}\right) \sigma(s)\right]\left[\sigma(r) \sigma\left(r^{\prime}\right)\right] z \\
& =\left[\sigma\left(r^{\prime}\right) \sigma(s)\right]\left[\sigma\left(r^{\prime}\right) \sigma(r)\right] z z \\
& =u_{\left(\sigma\left(r^{\prime}\right) \sigma(r) \sigma(s)\right)} \\
& =u_{\sigma\left(r s r^{\prime}\right) \sigma^{-1}}
\end{aligned}
$$

We now turn our attention to the Hochschild cohomology of $S_{-\mathbf{1}}(V) \#_{\alpha} S_{n}$.

Theorem 7.7 [Naidu and Witherspoon 2011, Theorem 6.8]. Assume that $n \geq 4$. The constant Hochschild 2-cocycles representing elements in $\mathrm{HH}^{2}\left(S_{-1}(V), S_{-1}(V) \# S_{n}\right)$ form a vector subspace of $\left(S_{-1}(V) \# G\right) \otimes \bigwedge_{-1}\left(V^{*}\right)$ having as a basis the set of all 


$$
\begin{array}{ll}
\eta_{1}=t_{1} \otimes v_{r}^{*} \wedge v_{s}^{*} & (r<s), \\
\eta_{2}=t_{(r s)} \otimes v_{r}^{*} \wedge v_{s}^{*} & (r<s), \\
\eta_{3}=t_{(r s)} \otimes\left(v_{r}^{*} \wedge v_{r^{\prime}}^{*}+v_{s}^{*} \wedge v_{r^{\prime}}^{*}\right) & (r<s), \\
\eta_{4}=t_{(r s)\left(r^{\prime} s^{\prime}\right)} \otimes\left(v_{r}^{*} \wedge v_{r^{\prime}}^{*}+v_{r}^{*} \wedge v_{s^{\prime}}^{*}+v_{s}^{*} \wedge v_{r^{\prime}}^{*}+v_{s}^{*} \wedge v_{s^{\prime}}^{*}\right) & \left(r<s, r^{\prime}<s^{\prime}, r<r^{\prime}\right), \\
\eta_{5}=t_{\left(r s r^{\prime}\right)} \otimes\left(v_{r}^{*} \wedge v_{s}^{*}+v_{s}^{*} \wedge v_{r^{\prime}}^{*}+v_{r}^{*} \wedge v_{r^{\prime}}^{*}\right) & \left(r<s, r<r^{\prime}\right) .
\end{array}
$$

Note that the $S_{-1}(V)$-bimodule structure of $S_{-1}(V) \#_{\alpha} G$ does not depend on the 2-cocycle $\alpha$, and so $\mathrm{HH}^{2}\left(S_{-1}(V), S_{-1}(V) \#_{\alpha} G\right)=\mathrm{HH}^{2}\left(S_{-1}(V), S_{-1}(V) \# G\right)$.

The lemma below involves the maps $\Theta_{2}^{*}, \mathscr{R}_{2}$, and $\Psi_{2}^{*}$ defined in Section 4. Recall that the image of an element $\sigma \in S_{n}$ in the twisted group algebra $\mathbb{C}^{\alpha} S_{n}$ is denoted by $t_{\sigma}$. Also, recall the definition of the 2-cocycle $\alpha$ given in (7.1).

Lemma 7.8. For all $i \neq j$,

$\left[\left(\Theta_{2}^{*} \mathscr{R}_{2} \Psi_{2}^{*}\right)\left(\eta_{a}\right)\right]\left(v_{i} \otimes v_{j}\right)$

$$
= \begin{cases}\frac{1}{n(n-1)} t_{1} & \text { if } a=1, \\ 0 & \text { if } a=2, \\ 0 & \text { if } a=3 \text { and } n \geq 5, \\ 0 & \text { if } a=4, \\ \frac{1}{n(n-1)(n-2)} \sum_{k \neq i, j}\left(2 t_{(i j k)}+t_{(i k j)}\right) & \text { if } a=5,\end{cases}
$$

Proof. Using (4.7),

$$
\begin{aligned}
{\left[\left(\Theta_{2}^{*} \Re_{2} \Psi_{2}^{*}\right)\left(\eta_{1}\right)\right]\left(v_{i} \otimes v_{j}\right) } & =\frac{1}{n !} \sum_{\sigma \in S_{n}} \sigma\left(\eta_{1}\left(\Psi_{2}\left(1 \otimes v_{\sigma^{-1}(i)} \otimes v_{\sigma^{-1}(j)} \otimes 1\right)\right)\right) \\
& =\frac{1}{n !} \sum_{\substack{\sigma \in S_{n} \\
\sigma^{-1}(i)<\sigma^{-1}(j)}} \sigma\left(\eta_{1}\left(1 \otimes 1 \otimes v_{\sigma^{-1}(i)} \otimes v_{\sigma^{-1}(j)}\right)\right) \\
& =\frac{1}{n !} \sum_{\substack{\sigma \in S_{n} \\
\sigma(r)=i, \sigma(s)=j}} \sigma\left(t_{1}\right) \\
& =\frac{1}{n(n-1)} t_{1} .
\end{aligned}
$$

Similarly,

$$
\left[\left(\Theta_{2}^{*} \mathscr{R}_{2} \Psi_{2}^{*}\right)\left(\eta_{2}\right)\right]\left(v_{i} \otimes v_{j}\right)=\frac{1}{n !} \sum_{\substack{\sigma \in S_{n} \\ \sigma(r)=i, \sigma(s)=j}} \sigma_{\left(t_{(r s)}\right)} .
$$

Applying the conjugation action in $\mathbb{C}^{\alpha} G$, we get 


$$
\frac{1}{n !} \sum_{\substack{\sigma \in S_{n} \\ \sigma(r)=i, \sigma(s)=j}} \frac{\alpha(\sigma,(r s))}{\alpha\left(\sigma(r s) \sigma^{-1}, \sigma\right)} t_{\sigma(r s) \sigma^{-1}}=\left(\frac{1}{n !} \sum_{\substack{\sigma \in S_{n} \\ \sigma(r)=i, \sigma(s)=j}} \frac{\alpha(\sigma,(r s))}{\alpha((i j), \sigma)}\right) t_{(i j)} \text {. }
$$

The scalar $\alpha(\sigma,(r s)) / \alpha((i j), \sigma)$ in the summation above is determined by the following element of $T_{n}$ :

$$
u_{\sigma} u_{(r s)} u_{\sigma(r s)}^{-1} u_{(i j) \sigma} u_{\sigma}^{-1} u_{(i j)}^{-1}=u_{\sigma} u_{(r s)} u_{\sigma}^{-1} u_{\sigma(r s) \sigma^{-1}}^{-1} .
$$

By Lemma 7.6(a),

$$
u_{\sigma} u_{(r s)} u_{\sigma}^{-1} u_{\sigma(r s) \sigma^{-1}}^{-1}= \begin{cases}z^{\epsilon(\sigma)} & \text { if } i<j \\ z^{\epsilon(\sigma)+1} & \text { if } i>j\end{cases}
$$

Since we assume $n$ is greater than or equal to 4 , the set $\left\{\sigma \in S_{n} \mid \sigma(r)=i, \sigma(s)=j\right\}$ contains an equal number of odd and even permutations, and so

$$
\sum_{\substack{\sigma \in S_{n} \\ \sigma(r)=i, \sigma(s)=j}} \frac{\alpha(\sigma,(r s))}{\alpha((i j), \sigma)}=0
$$

proving that $\left[\left(\Theta_{2}^{*} \mathscr{R}_{2} \Psi_{2}^{*}\right)\left(\eta_{2}\right)\right]\left(v_{i} \otimes v_{j}\right)=0$.

Next, we consider the $a=3$ case. In addition to the stated assumption $r<s$, assume further that $r<r^{\prime}$ and $s<r^{\prime}$. The other cases can be handled similarly. We have

$$
\left[\left(\Theta_{2}^{*} \mathscr{R}_{2} \Psi_{2}^{*}\right)\left(\eta_{3}\right)\right]\left(v_{i} \otimes v_{j}\right)=\frac{1}{n !} \sum_{\substack{\sigma \in S_{n} \\ \sigma(r)=i, \sigma\left(r^{\prime}\right)=j}} \sigma\left(t_{(r s)}\right)+\frac{1}{n !} \sum_{\substack{\sigma \in S_{n} \\ \sigma(s)=i, \sigma\left(r^{\prime}\right)=j}} \sigma\left(t_{(r s)}\right) .
$$

Applying the conjugation action in $\mathbb{C}^{\alpha} G$, we get

$$
\begin{aligned}
& \frac{1}{n !} \sum_{\substack{\sigma \in S_{n} \\
\sigma(r)=i, \sigma\left(r^{\prime}\right)=j}} \frac{\alpha(\sigma,(r s))}{\alpha((i \sigma(s)), \sigma)} t_{(i \sigma(s))}+\frac{1}{n !} \sum_{\substack{\sigma \in S_{n} \\
\sigma(s)=i, \sigma\left(r^{\prime}\right)=j}} \frac{\alpha(\sigma,(r s))}{\alpha((\sigma(r) i), \sigma)} t_{(\sigma(r) i)} \\
& \left.\quad=\frac{1}{n !} \sum_{\substack { k \neq i, j \\
\begin{subarray}{c}{\sigma \in S_{n} \\
\sigma(r)=i, \sigma\left(r^{\prime}\right)=j, \sigma(s)=k{ k \neq i , j \\
\begin{subarray} { c } { \sigma \in S _ { n } \\
\sigma ( r ) = i , \sigma ( r ^ { \prime } ) = j , \sigma ( s ) = k } }\end{subarray}} \frac{\alpha(\sigma,(r s))}{\alpha((i k), \sigma)}+\sum_{\substack{\sigma \in S_{n} \\
\sigma(s)=i, \sigma\left(r^{\prime}\right)=j, \sigma(r)=k}} \frac{\alpha(\sigma,(r s))}{\alpha((i k), \sigma)}\right) t_{(i k)} .
\end{aligned}
$$

The scalar $\alpha(\sigma,(r s)) / \alpha((i k), \sigma)$ in the first of the two inner summations above is determined by the element $u_{\sigma} u_{(r s)} u_{\sigma}^{-1} u_{\sigma(r s) \sigma^{-1}}^{-1}$ of $T_{n}$. Again, by Lemma 7.6(a),

$$
u_{\sigma} u_{(r s)} u_{\sigma}^{-1} u_{\sigma(r s) \sigma^{-1}}^{-1}= \begin{cases}z^{\epsilon(\sigma)} & \text { if } i<k \\ z^{\epsilon(\sigma)+1} & \text { if } i>k\end{cases}
$$

Since $n$ is assumed to be greater than or equal to 5, the set $\left\{\sigma \in S_{n} \mid \sigma(r)=i\right.$, $\left.\sigma\left(r^{\prime}\right)=j, \sigma(s)=k\right\}$ contains an equal number of odd and even permutations, and so

$$
\sum_{\substack{\sigma \in S_{n} \\ \sigma(r)=i, \sigma\left(r^{\prime}\right)=j, \sigma(s)=k}} \frac{\alpha(\sigma,(r s))}{\alpha((i k), \sigma)}=0 .
$$


Similarly,

$$
\sum_{\substack{\sigma \in S_{n} \\ \sigma(s)=i, \sigma\left(r^{\prime}\right)=j, \sigma(r)=k}} \frac{\alpha(\sigma,(r s))}{\alpha((i k), \sigma)}=0,
$$

and it follows that $\left[\left(\Theta_{2}^{*} \mathscr{R}_{2} \Psi_{2}^{*}\right)\left(\eta_{3}\right)\right]\left(v_{i} \otimes v_{j}\right)=0$.

For the $a=4$ case, in addition to the stated assumptions $r<s, r^{\prime}<s^{\prime}, r<r^{\prime}$, assume further that $r<s^{\prime}, s<r^{\prime}$, and $s<s^{\prime}$. The other cases can be handled similarly. We have $\left[\left(\Theta_{2}^{*} \mathscr{R}_{2} \Psi_{2}^{*}\right)\left(\eta_{4}\right)\right]\left(v_{i} \otimes v_{j}\right)$

$$
\begin{aligned}
& =\frac{1}{n !} \sum_{\substack{\sigma \in S_{n} \\
\sigma(r)=i, \sigma\left(r^{\prime}\right)=j}}{ }^{\sigma}\left(t_{(r s)\left(r^{\prime} s^{\prime}\right)}\right)+\frac{1}{n !} \sum_{\substack{\sigma \in S_{n} \\
\sigma(r)=i, \sigma\left(s^{\prime}\right)=j}} \sigma_{\left(r_{(r s)\left(r^{\prime} s^{\prime}\right)}\right)} \\
& +\frac{1}{n !} \sum_{\substack{\sigma \in S_{n} \\
\sigma(s)=i, \sigma\left(r^{\prime}\right)=j}} \sigma\left(t_{(r s)\left(r^{\prime} s^{\prime}\right)}\right)+\frac{1}{n !} \sum_{\substack{\sigma \in S_{n} \\
\sigma(s)=i, \sigma\left(s^{\prime}\right)=j}}{ }\left(t_{(r s)\left(r^{\prime} s^{\prime}\right)}\right) .
\end{aligned}
$$

Applying the conjugation action in $\mathbb{C}^{\alpha} G$, we get

$$
\begin{aligned}
& \frac{1}{n !}\left(\sum_{\sigma \in S_{n}} \frac{\alpha\left(\sigma,(r s)\left(r^{\prime} s^{\prime}\right)\right)}{\alpha\left((i \sigma(s))\left(j \sigma\left(s^{\prime}\right)\right), \sigma\right)} t_{(i \sigma(s))\left(j \sigma\left(s^{\prime}\right)\right)}\right. \\
& \sigma(r)=i, \sigma\left(r^{\prime}\right)=j+\sum_{\sigma \in S_{n}} \frac{\alpha\left(\sigma,(r s)\left(r^{\prime} s^{\prime}\right)\right)}{\alpha\left((i \sigma(s))\left(\sigma\left(r^{\prime}\right) j\right), \sigma\right)} t_{(i \sigma(s))\left(\sigma\left(r^{\prime}\right) j\right)} \\
& \sigma(r)=i, \sigma\left(s^{\prime}\right)=j \\
& +\sum_{\sigma \in S_{n}} \frac{\alpha\left(\sigma,(r s)\left(r^{\prime} s^{\prime}\right)\right)}{\alpha\left((\sigma(r) i)\left(j \sigma\left(s^{\prime}\right)\right), \sigma\right)} t_{(\sigma(r) i)\left(j \sigma\left(s^{\prime}\right)\right)} \\
& \sigma(s)=i, \sigma\left(r^{\prime}\right)=j \\
& \left.+\sum_{\sigma \in S_{n}} \frac{\alpha\left(\sigma,(r s)\left(r^{\prime} s^{\prime}\right)\right)}{\alpha\left((\sigma(r) i)\left(\sigma\left(r^{\prime}\right) j\right), \sigma\right)} t_{(\sigma(r) i)\left(\sigma\left(r^{\prime}\right) j\right)}\right) \\
& \sigma(s)=i, \sigma\left(s^{\prime}\right)=j
\end{aligned}
$$

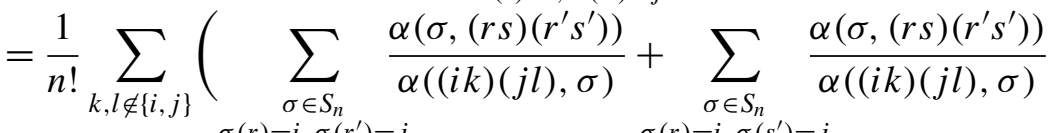

$$
\begin{aligned}
& \begin{array}{ll}
\sigma(r)=i, \sigma\left(r^{\prime}\right)=j & \sigma(r)=i, \sigma\left(s^{\prime}\right)=j \\
\sigma(s)=k, \sigma\left(s^{\prime}\right)=l & \sigma(s)=k, \sigma\left(r^{\prime}\right)=l
\end{array} \\
& \left.+\sum_{\sigma \in S_{n}} \frac{\alpha\left(\sigma,(r s)\left(r^{\prime} s^{\prime}\right)\right)}{\alpha((i k)(j l), \sigma)}+\sum_{\sigma \in S_{n}} \frac{\alpha\left(\sigma,(r s)\left(r^{\prime} s^{\prime}\right)\right)}{\alpha((i k)(j l), \sigma)}\right) t_{(i k)(j l)} . \\
& \sigma(s)=i, \sigma\left(r^{\prime}\right)=j \\
& \sigma(r)=k, \sigma\left(s^{\prime}\right)=l \\
& \sigma(s)=i, \sigma\left(s^{\prime}\right)=j \\
& \sigma(r)=k, \sigma\left(r^{\prime}\right)=l
\end{aligned}
$$

The scalar $\alpha\left(\sigma,(r s)\left(r^{\prime} s^{\prime}\right)\right) / \alpha((i k)(j l), \sigma)$ in the first of the four inner summations above is determined by the element $u_{\sigma} u_{(r s)\left(r^{\prime} s^{\prime}\right)} u_{\sigma}^{-1} u_{\sigma(r s)\left(r^{\prime} s^{\prime}\right) \sigma^{-1}}^{-1}$ of $T_{n}$. By Lemma 7.6(b), 


$$
u_{\sigma} u_{(r s)\left(r^{\prime} s\right)} u_{\sigma}^{-1} u_{\sigma(r s)\left(r^{\prime} s^{\prime}\right) \sigma^{-1}}^{-1}=z^{d_{\sigma}\left(r, s, r^{\prime} s^{\prime}\right)}=z^{d(i, k, j, l)}
$$

Thus,

$$
\sum_{\substack{\sigma \in S_{n} \\ \sigma(r)=i, \sigma\left(r^{\prime}\right)=j \\ \sigma(s)=k, \sigma\left(s^{\prime}\right)=l}} \frac{\alpha\left(\sigma,(r s)\left(r^{\prime} s^{\prime}\right)\right)}{\alpha((i k)(j l), \sigma)}=(n-4) !(-1)^{d(i, k, j, l)} .
$$

Similarly, the second, third, and fourth summations are equal to $(n-1)$ ! times $(-1)^{d(i, k, l, j)},(-1)^{d(k, i, j, l)}$, and $(-1)^{d(k, i, l, j)}$, respectively. From Lemma 7.5 it follows that the sum of the four summations above is equal to zero, and so $\left[\left(\Theta_{2}^{*} \mathscr{R}_{2} \Psi_{2}^{*}\right)\left(\eta_{4}\right)\right]\left(v_{i} \otimes v_{j}\right)=0$.

Finally, for the $a=5$ case, in addition to the stated assumptions $r<s, r<r^{\prime}$, assume further that $s<r^{\prime}$. Again, the other case can be handled similarly. We have $\left[\left(\Theta_{2}^{*} \mathscr{R}_{2} \Psi_{2}^{*}\right)\left(\eta_{5}\right)\right]\left(v_{i} \otimes v_{j}\right)$

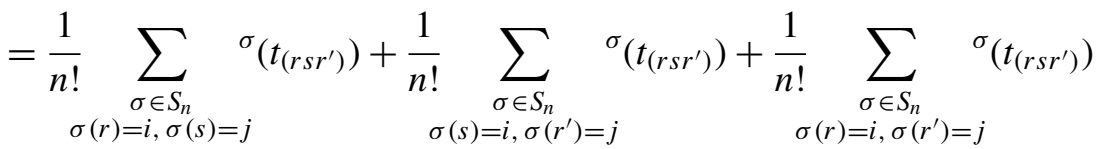

$$
\begin{aligned}
& =\frac{1}{n !} \sum_{\substack{\sigma \in S_{n} \\
\sigma(r)=i, \sigma(s)=j}} \frac{\alpha\left(\sigma,\left(r s r^{\prime}\right)\right)}{\alpha\left(\left(i j \sigma\left(r^{\prime}\right)\right), \sigma\right)} t_{\left(i j \sigma\left(r^{\prime}\right)\right)}+\frac{1}{n !} \sum_{\substack{\sigma \in S_{n} \\
\sigma(s)=i, \sigma\left(r^{\prime}\right)=j}} \frac{\alpha\left(\sigma,\left(r s r^{\prime}\right)\right)}{\alpha((\sigma(r) i j), \sigma)} t_{(\sigma(r) i j)} \\
& +\frac{1}{n !} \sum_{\substack{\sigma \in S_{n} \\
\sigma(r)=i, \sigma\left(r^{\prime}\right)=j}} \frac{\alpha\left(\sigma,\left(r s r^{\prime}\right)\right)}{\alpha((i \sigma(s) j), \sigma)} t_{(i \sigma(s) j)} \\
& =\frac{1}{n !} \sum_{k, l \notin\{i, j\}}\left[\left(\sum_{\substack{\sigma \in S_{n} \\
\sigma(r)=i, \sigma(s)=j, \sigma\left(r^{\prime}\right)=k}} \frac{\alpha\left(\sigma,\left(r s r^{\prime}\right)\right)}{\alpha((i j k), \sigma)}+\sum_{\substack{\sigma \in S_{n} \\
\sigma(s)=i, \sigma\left(r^{\prime}\right)=j, \sigma(r)=k}} \frac{\alpha\left(\sigma,\left(r s r^{\prime}\right)\right)}{\alpha((i j k), \sigma))}\right) t_{(i j k)}\right. \\
& \left.+\quad \sum_{\sigma \in S_{n}} \frac{\alpha\left(\sigma,\left(r s r^{\prime}\right)\right)}{\alpha((i k j), \sigma)} t_{(i k j)}\right] \text {. } \\
& \sigma(r)=i, \sigma\left(r^{\prime}\right)=j, \sigma(s)=k
\end{aligned}
$$

The scalar $\alpha\left(\sigma,\left(r s r^{\prime}\right)\right) / \alpha((i j k), \sigma)$ in the first of the three inner summations above is determined by the element $u_{\sigma} u_{\left(r s r^{\prime}\right)} u_{\sigma}^{-1} u_{\sigma\left(r s r^{\prime}\right) \sigma^{-1}}^{-1}$ of $T_{n}$. By Lemma 7.6(c), $u_{\sigma} u_{\left(r s r^{\prime}\right)} u_{\sigma}^{-1} u_{\sigma\left(r s r^{\prime}\right) \sigma^{-1}}^{-1}=1$. Thus,

$$
\sum_{\substack{\sigma \in S_{n} \\ \sigma(r)=i, \sigma(s)=j \sigma\left(r^{\prime}\right)=k}} \frac{\alpha\left(\sigma,\left(r s r^{\prime}\right)\right.}{\alpha((i j k), \sigma)}=(n-3) ! .
$$

Similarly, the second and third summations are also equal to $(n-3) !$. It follows that

$$
\left[\left(\Theta_{2}^{*} \mathscr{R}_{2} \Psi_{2}^{*}\right)\left(\eta_{5}\right)\right]\left(v_{i} \otimes v_{j}\right)=\frac{1}{n(n-1)(n-2)} \sum_{k \neq i, j}\left(2 t_{(i j k)}+t_{(i k j)}\right) .
$$


Combining Theorems 7.7, 4.4, 5.4, and Lemma 7.8 establishes the following.

Theorem 7.9. Assume that $n \geq 5$. The maps $\kappa: V \times V \rightarrow \mathbb{C}^{\alpha} S_{n}$ for which $\mathscr{H}_{-\mathbf{1}, \kappa, \alpha}$ is a twisted quantum Drinfeld Hecke algebra form a two-dimensional vector space with basis consisting of bilinear maps $\kappa_{1}: V \times V \rightarrow \mathbb{C}^{\alpha} S_{n}$ and $\kappa_{2}: V \times V \rightarrow \mathbb{C}^{\alpha} S_{n}$ determined by

$$
\kappa_{1}\left(v_{i}, v_{j}\right)=t_{1} \quad \text { and } \quad \kappa_{2}\left(v_{i}, v_{j}\right)=\sum_{k \neq i, j}\left(t_{(i j k)}+t_{(i k j)}\right)
$$

for all $i \neq j$.

\section{References}

[Adem and Ruan 2003] A. Adem and Y. Ruan, "Twisted orbifold K-theory", Comm. Math. Phys. 237:3 (2003), 533-556. MR 2004e:19004 Zbl 1051.57022

[Bazlov and Berenstein 2009] Y. Bazlov and A. Berenstein, "Noncommutative Dunkl operators and braided Cherednik algebras", Selecta Math. (N.S.) 14:3-4 (2009), 325-372. MR 2010k:16044 Zbl 1220.16027

[Bergman 1978] G. M. Bergman, "The diamond lemma for ring theory", Adv. in Math. 29:2 (1978), 178-218. MR 81b:16001 Zbl 0326.16019

[Căldăraru et al. 2004] A. Căldăraru, A. Giaquinto, and S. Witherspoon, "Algebraic deformations arising from orbifolds with discrete torsion”, J. Pure Appl. Algebra 187:1-3 (2004), 51-70. MR 2005c:16013 Zbl 1055.16010

[Chmutova 2005] T. Chmutova, "Twisted symplectic reflection algebras", preprint, 2005. arXiv math/ 0505653

[Drinfeld 1986] V. G. Drinfeld, "Degenerate affine Hecke algebras and Yangians", Funktsional. Anal. i Prilozhen. 20:1 (1986), 69-70. In Russian; translated in Funct. Anal. Appl. 20 (1986), 58-60. MR 87m:22044 Zbl 0599.20049

[Etingof and Ginzburg 2002] P. Etingof and V. Ginzburg, "Symplectic reflection algebras, CalogeroMoser space, and deformed Harish-Chandra homomorphism", Invent. Math. 147:2 (2002), 243-348. MR 2003b:16021 Zbl 1061.16032

[Gordon 2003] I. Gordon, "On the quotient ring by diagonal invariants", Invent. Math. 153:3 (2003), 503-518. MR 2004f:20075 Zbl 1039.20019

[Levandovskyy and Shepler 2011] V. Levandovskyy and A. Shepler, "Quantum Drinfeld Hecke algebras", preprint, 2011. arXiv 1111.4975

[Lusztig 1989] G. Lusztig, "Affine Hecke algebras and their graded version”, J. Amer. Math. Soc. 2:3 (1989), 599-635. MR 90e:16049 Zbl 0715.22020

[Naidu and Witherspoon 2011] D. Naidu and S. Witherspoon, "Hochschild cohomology and quantum Drinfeld Hecke algebras", preprint, 2011. arXiv 1111.5243

[Naidu et al. 2011] D. Naidu, P. Shroff, and S. Witherspoon, "Hochschild cohomology of group extensions of quantum symmetric algebras", Proc. Amer. Math. Soc. 139:5 (2011), 1553-1567. MR 2012b:16026 Zbl 1259.16011

[Ram and Shepler 2003] A. Ram and A. V. Shepler, "Classification of graded Hecke algebras for complex reflection groups", Comment. Math. Helv. 78:2 (2003), 308-334. MR 2004d:20007 Zbl 1063.20005 
[Schur 2001] J. Schur, "On the representation of the symmetric and alternating groups by fractional linear substitutions", Internat. J. Theoret. Phys. 40:1 (2001), 413-458. MR 2003a:20016 Zbl 0969.20002

[Shepler and Witherspoon 2012] A. V. Shepler and S. Witherspoon, "Group actions on algebras and the graded Lie structure of Hochschild cohomology”, J. Algebra 351 (2012), 350-381. MR 2862214 Zbl 06046981

[Ştefan 1995] D. Ştefan, "Hochschild cohomology on Hopf Galois extensions", J. Pure Appl. Algebra 103:2 (1995), 221-233. MR 96h:16013 Zbl 0838.16008

[Vafa and Witten 1995] C. Vafa and E. Witten, "On orbifolds with discrete torsion", J. Geom. Phys. 15:3 (1995), 189-214. MR 95m:81190 Zbl 0816.53053

[Vendramin 2012] L. Vendramin, "Nichols algebras associated to the transpositions of the symmetric group are twist-equivalent”, Proc. Amer. Math. Soc. 140:11 (2012), 3715-3723. MR 2944712

[Wambst 1993] M. Wambst, "Complexes de Koszul quantiques", Ann. Inst. Fourier (Grenoble) 43:4 (1993), 1089-1156. MR 95a:17023 Zbl 0810.16010

[Witherspoon 2007] S. Witherspoon, “Twisted graded Hecke algebras”, J. Algebra 317:1 (2007), 30-42. MR 2009a:20009 Zbl 1139.20005

Received October 24, 2012.

DEEPAK NAIDU

DEPARTMENT OF MATHEMATICAL SCIENCES

NORTHERN ILLINOIS UNIVERSITY

DEKALB, ILLINOIS 60115

UNITED STATES

dnaidu@math.niu.edu 


\title{
PACIFIC JOURNAL OF MATHEMATICS
}

\author{
msp.org/pjm
}

Founded in 1951 by E. F. Beckenbach (1906-1982) and F. Wolf (1904-1989)

\section{EDITORS}

V. S. Varadarajan (Managing Editor)

Department of Mathematics

University of California

Los Angeles, CA 90095-1555

pacific@math.ucla.edu

Paul Balmer

Department of Mathematics

University of California

Los Angeles, CA 90095-1555

balmer@math.ucla.edu

Daryl Cooper

Department of Mathematics

University of California

Santa Barbara, CA 93106-3080 cooper@math.ucsb.edu

Jiang-Hua $\mathrm{Lu}$

Department of Mathematics

Pokfulam Rd., Hong Kong jhlu@maths.hku.hk
The University of Hong Kong

Don Blasius

Department of Mathematics University of California

Los Angeles, CA 90095-1555

blasius@math.ucla.edu

Robert Finn

Department of Mathematics Stanford University

Stanford, CA 94305-2125

finn@math.stanford.edu

Sorin Popa

Department of Mathematics

University of California

Los Angeles, CA 90095-1555 popa@math.ucla.edu

Paul Yang

Department of Mathematics Princeton University

Princeton NJ 08544-1000

yang@math.princeton.edu

\section{PRODUCTION}

Silvio Levy, Scientific Editor, production@msp.org

\section{SUPPORTING INSTITUTIONS}

ACADEMIA SINICA, TAIPEI

CALIFORNIA INST. OF TECHNOLOGY

INST. DE MATEMÁTICA PURA E APLICADA

KEIO UNIVERSITY

MATH. SCIENCES RESEARCH INSTITUTE

NEW MEXICO STATE UNIV.

OREGON STATE UNIV.

\author{
STANFORD UNIVERSITY \\ UNIV. OF BRITISH COLUMBIA \\ UNIV. OF CALIFORNIA, BERKELEY \\ UNIV. OF CALIFORNIA, DAVIS \\ UNIV. OF CALIFORNIA, LOS ANGELES \\ UNIV. OF CALIFORNIA, RIVERSIDE \\ UNIV. OF CALIFORNIA, SAN DIEGO \\ UNIV. OF CALIF., SANTA BARBARA
}

\author{
Vyjayanthi Chari \\ Department of Mathematics \\ University of California \\ Riverside, CA 92521-0135 \\ chari@math.ucr.edu \\ Kefeng Liu \\ Department of Mathematics \\ University of California \\ Los Angeles, CA 90095-1555 \\ liu@math.ucla.edu \\ Jie Qing \\ Department of Mathematics \\ University of California \\ Santa Cruz, CA 95064 \\ qing@cats.ucsc.edu
}

These supporting institutions contribute to the cost of publication of this Journal, but they are not owners or publishers and have no responsibility for its contents or policies.

See inside back cover or msp.org/pjm for submission instructions.

The subscription price for 2014 is US \$410/year for the electronic version, and \$535/year for print and electronic.

Subscriptions, requests for back issues and changes of subscribers address should be sent to Pacific Journal of Mathematics, P.O. Box 4163, Berkeley, CA 94704-0163, U.S.A. The Pacific Journal of Mathematics is indexed by Mathematical Reviews, Zentralblatt MATH, PASCAL CNRS Index, Referativnyi Zhurnal, Current Mathematical Publications and Web of Knowledge (Science Citation Index).

The Pacific Journal of Mathematics (ISSN 0030-8730) at the University of California, c/o Department of Mathematics, 798 Evans Hall \#3840, Berkeley, CA 94720-3840, is published twelve times a year. Periodical rate postage paid at Berkeley, CA 94704, and additional mailing offices. POSTMASTER: send address changes to Pacific Journal of Mathematics, P.O. Box 4163, Berkeley, CA 94704-0163.

PJM peer review and production are managed by EditFLOW ${ }^{\circledR}$ from Mathematical Sciences Publishers.

\section{PUBLISHED BY}

mathematical sciences publishers

nonprofit scientific publishing

http://msp.org/

(C) 2014 Mathematical Sciences Publishers 


\section{PACIFIC JOURNAL OF MATHEMATICS}

Volume $268 \quad$ No. $1 \quad$ March 2014

AlEXANDRE PAIVA BARRETO

A transport inequality on the sphere obtained by mass transport

DARIO CORDERO-ERAUSQUIN

A cohomological injectivity result for the residual automorphic spectrum of $\mathrm{GL}_{n}$

HARALD GROBNER

Gradient estimates and entropy formulae of porous medium and fast diffusion equations for the Witten Laplacian

GUANGYUE HUANG and HAIZHONG LI

Controlled connectivity for semidirect products acting on locally finite trees

KeITH JoNES

An indispensable classification of monomial curves in $\mathbb{A}^{4}(\mathbb{k})$

ANARGYROS KATSABEKIS and IGNACIO OJEDA

Contracting an axially symmetric torus by its harmonic mean curvature

CHRISTOPHER KIM

Composition operators on strictly pseudoconvex domains with smooth symbol

HYUNGWOON KOO and SONG-YING LI

The Alexandrov problem in a quotient space of $\mathbb{U}^{2} \times \mathbb{R}$

AnA Menezes

Twisted quantum Drinfeld Hecke algebras

DEEPAK NAIDU

$L^{p}$ harmonic 1-forms and first eigenvalue of a stable minimal hypersurface

KEOMKYO SEO

Reconstruction from Koszul homology and applications to module and derived categories

RYO TAKAHASHI

A virtual Kawasaki-Riemann-Roch formula 